\title{
亚砜化合物的生物活性研究和不对称合成进展
}

\author{
朱海梦 $a$ 王 超*,b宗利利 $*, a$ \\ ( $a$ 厦门大学药学院 厦门 361102) \\ ( $b$ 南京工业大学先进化学制造研究院 南京 211816)
}

\begin{abstract}
摘要 亚砜类化合物在有机合成和药物化学中有着广泛的应用，手性亚砜作为助剂、配体、催化剂和合成子的用途已 得到充分证明，亚砜类化合物的亚磺酰基团也可作为药效团、羰基生物等排体或药物分子修饰基团应用于药物设计和 药物开发之中. 综述了具有生物活性的亚砜类化合物的结构骨架以及相应的作用机制, 并总结了近十年来通过构建硫 立体中心制备手性亚砜类化合物的新方法和新进展，具体到廉价金属铁络合物、多金属氧酸盐、有机小分子、生物酶 和电化学催化的硫醚不对称氧化反应，以及基于次磺酸阴离子中间体的手性亚砜合成策略和最近涌现的新策略，其中 也包括作者最近在手性亚砜合成领域取得一些研究成果.
\end{abstract}

关键词 亚砜生物活性分子; 亚砜药物; 不对称合成; 硫醚氧化反应; 次磺酸阴离子; 多金属氧酸盐

\section{Progress on Biological Activity Study and Enantioselective Synthesis of Sulfoxides}

\author{
Zhu, Haimeng ${ }^{a} \quad$ Wang, Chao ${ }^{*, b}$ Zong, Lili ${ }^{*, a}$ \\ ( ${ }^{a}$ School of Pharmaceutical Sciences, Xiamen University, Xiamen 361102) \\ ( ${ }^{b}$ Institute of Advanced Synthesis, Nanjing Tech University, Nanjing 211816)
}

\begin{abstract}
Sulfoxide compounds bearing unique sulfinyl group have attracted extensive attentions due to their important application in asymmetric organic synthesis and medicinal chemistry. The utility of chiral sulfoxides as versatile auxiliaries, ligands, and catalysts and synthons has been well demonstrated. Furthermore, the sulfinyl moiety can act as a pharmaceutical core, a bioisosteric replacement of the carbonyl moiety or a modifier in drug design and drug development. In this review, their biological activities with esomeprazole, ajoene, sulforaphane, sulforaphene, cenicriviroc, arbidol sulfoxide, armodafinil and so on are exemplified. And their mechanisms of action are illustrated briefly. Since the sulfur chirality is different from carbon stereogenic center, much effort has been devoted to the preparation of chiral sulfoxides. Herein the advances in asymmetric synthesis of sulfoxide during the past decade are presented. The direct asymmetric oxidation of prochiral sulfides with cheap iron-complex catalyst, polyoxometalates, organocatalyst, biocatalyst and electrocatalyst is discussed. Moreover, the complementary strategy based on sulfenate anions and miscellaneous strategies emerged recently are described. Additionally, our recent research works in the construction of chiral sulfoxide are also mentioned.
\end{abstract}

Keywords bioactive sulfoxide; sulfoxide drug; asymmetric synthesis; sulfoxidation; sulfenate anion; polyoxometalates

在亚砜类化合物 $\left(\mathrm{R}^{1} \mathrm{~S}(\mathrm{O}) \mathrm{R}^{2}\right)$ 中, 亚磺酰基的 $\mathrm{SO}$ 键 在强度和键长等方面具有双键的特征, 但是, 与羰基 $(\mathrm{C}=\mathrm{O})$ 和烯基 $(\mathrm{C}=\mathrm{C})$ 的双键相比, SO 键又具有独特的 成键方式、结构特征以及化学性质. 例如, $\mathrm{SO}$ 键的 $\mathrm{S}$ 原 子为 $\mathrm{sp}^{3}$ 杂化, 且 $\mathrm{S}$ 和 $\mathrm{O}$ 原子均具有孤对电子, 易于与 金属离子络合形成配合物; $\mathrm{S}$ 原子中心呈金字塔形(图 $1 a)$, 当 $R^{1} \neq R^{2}$ 时, $S$ 原子中心是手性中心, 因此手性亚砜
化合物具有光学活性; 亚磺酰基可作为吸电子基团活化 $\alpha$-亚甲基，使其在碱性条件下易于脱去质子生成亲核试 剂, 进而参与一系列反应. 因此, 亚砜尤其是手性亚砜 是一类用途十分广泛的化合物，可作为配体如 $1 \sim \mathbf{5}^{[1-7]}$ 、 催化剂、助剂[8]以及合成子[9-10]等(图 1b). 例如中国科学 院成都生物研究所 Liao 课题组开发的双叔丁基手性亚 砜配体 ${ }^{[11]}$ 以及磷-手性亚砜配体 $\mathbf{4}^{[4]}$, 在多种不对称催化

\footnotetext{
* Corresponding authors. E-mail: iasc.wang@njtech.edu.cn; lili.zong@xmu.edu.cn Received March 25, 2021; revised April 28, 2021; published online June 15, 2021. Project supported by the National Natural Science Foundation of China (No. 21802018) and the Fujian Provincial Joint Research Project (No. 2020I0003). 国家自然科学基金(No. 21802018)和福建省对外合作(No. 2020I0003)资助项目.
} 
反应展示了优异的立体控制能力. 值得一提的是, 某些

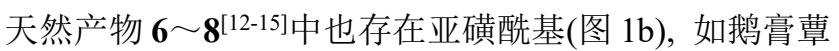
碱 $\mathbf{8}^{[16-18]}$, 它是一种二环八肽, 具有高致死毒性 $\left(\mathrm{LD}_{50}=\right.$ $50 \sim 100 \mu \mathrm{g} / \mathrm{kg}$ ), 是 RNA 聚合酶 II 的高选择性变构抑制 剂. 此外, 亚磺酰基是羰基的生物等排体 ${ }^{[19]}$, 在药物结 构设计和改造中也有着广泛的应用, 例如可用于提高药 物分子的水溶性, 影响药物代谢动力学性质, 增强药物 与靶标蛋白的相互结合能力等.

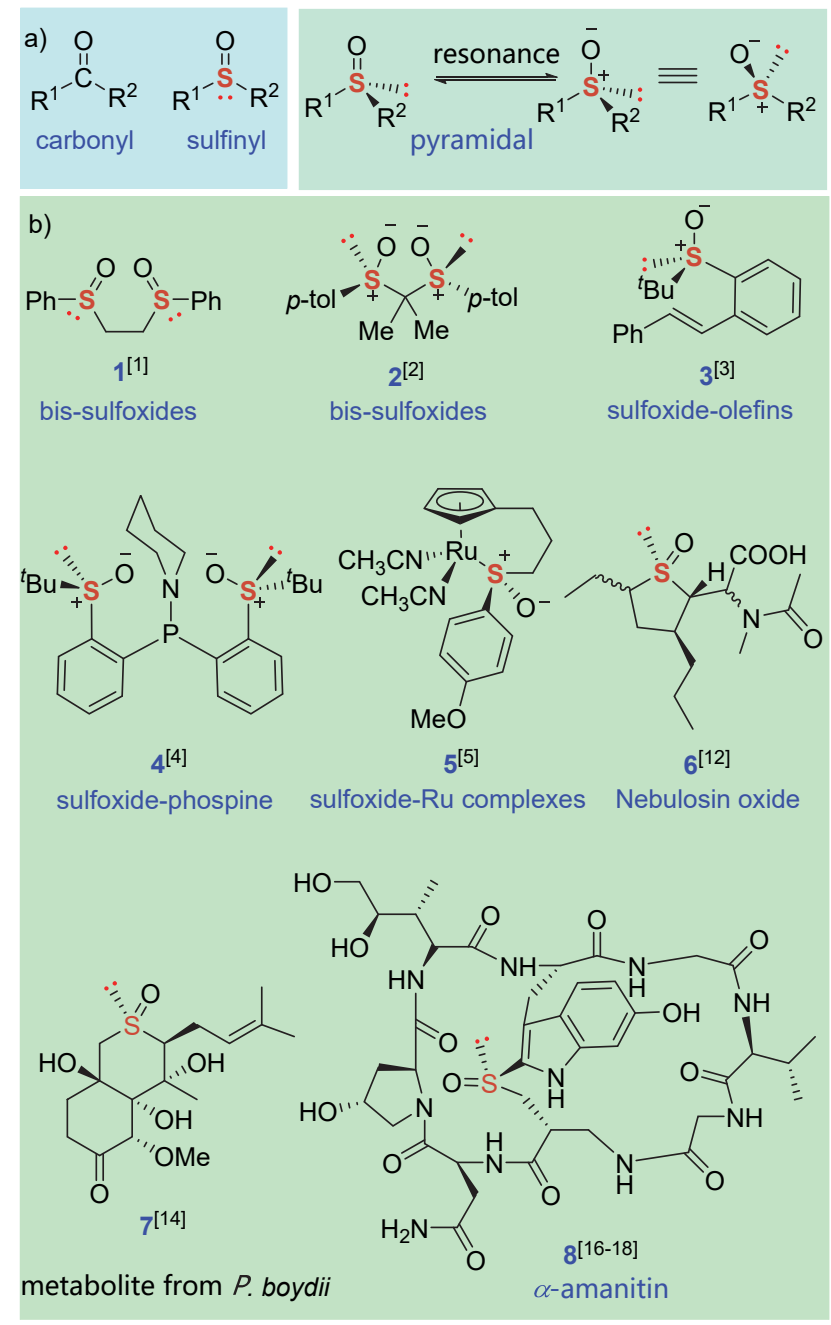

图 1 亚砜类化合物的结构和应用

Figure 1 Structure and application of sulfoxides

本文主要综述几类典型的生物活性亚砜化合物的 结构骨架和药物应用, 并结合自己的研究工作对手性亚 砜不对称合成领域的重要进展做简要论述.

\section{1 亚砜类化合物的生物活性}

\section{1 苯并咪唑类亚砜衍生物}

亚砜类手性药物埃索美拉唑(Esomeprazole, 10)成 功入围 2019 年全球药品销售榜前 200 强, 以 22.23 亿美 元的年销售额位居榜单第 58 位. 埃索美拉唑是一种胃
肠类药物, 能够有效地治疗因胃酸分泌过多而引发的相 关疾病，例如胃溃疡、十二指肠溃疡和胃食管反流病 (Gastroesophageal reflux disease, GERD). 埃索美拉唑是 首个被应用于临床的质子泵抑制剂(Proton pump inhibitor, PPI) 奥美拉唑 9 的 $(S)$-单一对映体(图 2), 其结构中含 有苯并咪唑环和多取代吡啶骨架以及一个硫原子手性 中心，比外消旋体奥美拉唑具有更高的生物利用度和更 少的副作用. 对奥美拉唑进行结构修饰和微调, 人们得 到了一系列 PPI 类药物, 例如具有咪唑并吡啶骨架的泰 妥拉唑(Tenatoprazol, 11)、含氟原子的泮托拉唑 (Pantoprazole, 12)和兰索拉唑(Lansoprazole, 13)、雷贝拉唑 (Rabeprazole, 14)、含有吡咯的艾普拉唑(Ilaprazole, 15) 以及含有 $N$-取代苯胺基的莱米诺拉唑(Leminoprazole, 16)等.

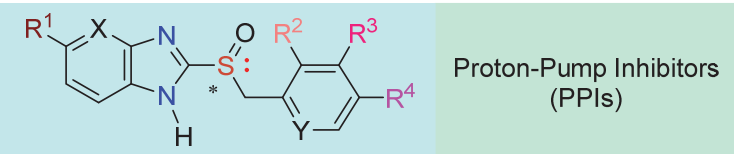

9 Omeprazole: $\mathrm{X}=\mathrm{CH}, \mathrm{Y}=\mathrm{N}, \mathrm{R}^{1}=\mathrm{R}^{3}=\mathrm{OMe}, \mathrm{R}^{2}=\mathrm{R}^{4}=\mathrm{Me}$ 10 Esomeprazole: $(S)$-Omeprazole;

11 Tenatoprazole: $X=N, Y=N, R^{1}=R^{3}=O M e, R^{2}=R^{4}=M e$; 12 Pantoprazole: $X=\mathrm{CH}, \mathrm{Y}=\mathrm{N}, \mathrm{R}^{1}=\mathrm{OCF}_{2} \mathrm{H}, \mathrm{R}^{2}=\mathrm{R}^{3}=\mathrm{OCH}_{3}$, $\mathrm{R}^{4}=\mathrm{H}$; 13 Lansoprazole: $\mathrm{X}=\mathrm{CH}, \mathrm{Y}=\mathrm{N}, \mathrm{R}^{1}=\mathrm{R}^{4}=\mathrm{H}, \mathrm{R}^{2}=\mathrm{Me}$, $\mathrm{R}^{3}=\mathrm{OCH}_{2} \mathrm{CF}_{3}$; 14 Rabeprazole: $\mathrm{X}=\mathrm{CH}, \mathrm{Y}=\mathrm{N}, \mathrm{R}^{1}=\mathrm{R}^{4}=\mathrm{H}$, $\mathrm{R}^{2}=\mathrm{Me}, \mathrm{R}^{3}=\mathrm{O}\left(\mathrm{CH}_{2}\right)_{3} \mathrm{OMe}$; 15 Ilaprazole: $\mathrm{X}=\mathrm{CH}, \mathrm{Y}=\mathrm{N}, \mathrm{R}^{1}=$ $1 H$-pyrrol, $\mathrm{R}^{2}=\mathrm{Me}, \mathrm{R}^{3}=\mathrm{OMe}, \mathrm{R}^{4}=\mathrm{H} ; 16$ Leminoprazole: $\mathrm{X}=$ $\mathrm{CH}, \mathrm{Y}=\mathrm{CNMe} \mathrm{B}^{j} \mathrm{Bu}, \mathrm{R}^{1}=\mathrm{R}^{2}=\mathrm{R}^{3}=\mathrm{R}^{4}=\mathrm{H}$

图 2 亚砜类质子原抑制剂的结构式

Figure 2 Structures of sulfoxides-type proton pump inhibitors

值得注意的是，以上所述的亚砜类药物均隶属于小 分子共价抑制剂. 以埃索美拉唑(10)为例，它的次磺酸 或次磺酰胺代谢物可以通过二硫键与分布于胃部壁细 胞分泌膜上的质子泵膜蛋白 $\left(\mathrm{H}^{+} \mathrm{K}^{+}\right.$-ATPase $)$发生共价键 结合(Scheme 1), 使此类在胃酸分泌过程起关键作用的 质子洜膜蛋白失去其转运功能, 从而阻止了胃酸的过量 分泌，进而发挥其治疗胃酸分泌失调相关疾病的功效.

另外, 研究发现, 兰索拉唑 13 及其衍生物与可能导 致阿尔兹海默症等神经退行性疾病的 Tau 神经原纤维缠 结(Neurofibrillary tangles, NFTs), 具有较高的体外亲合 力 $\left(\mathrm{IC}_{50}: 10^{-8} \sim 10^{-10} \mathrm{~mol} / \mathrm{L}\right)^{[20]}$. 因此, $\left[{ }^{18} \mathrm{~F}\right]$-兰索拉唑及 其衍生物可作为正电子发射计算机断层显像技术 (Positron emission tomography, PET)的潜在放射性示踪 剂, 有助于此类疾病的早期发现、早期诊断和精准治疗.

\section{2 阿焦烯}

新鲜大蒜被压碎、切碎或咀嚼时, 在自身所含蒜氨 酸裂解酶的作用下，其中的蒜氨酸(Alliin，17)将转化成 不稳定的蒜素(Allicin), 之后蒜素通过重排反应可以转 


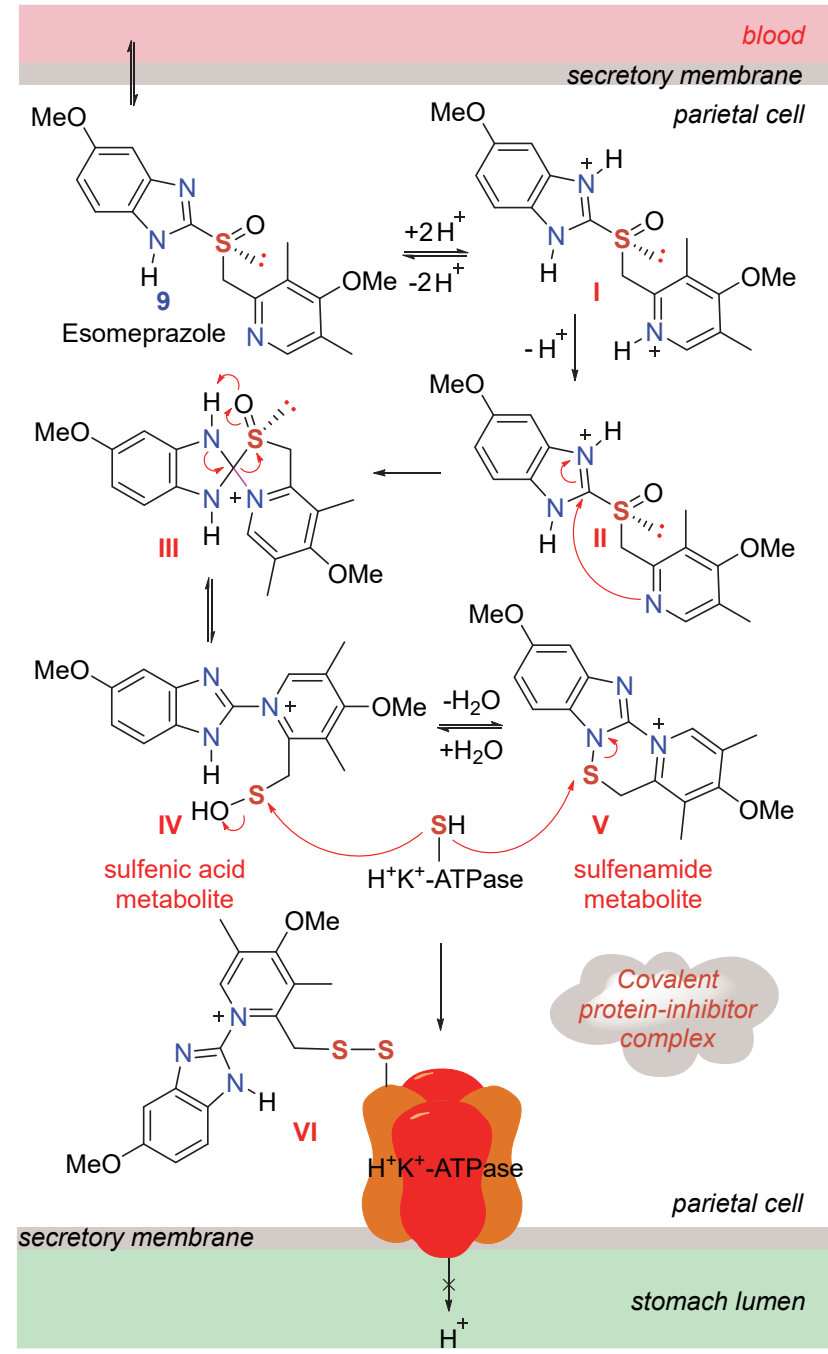

图式 1 埃索美拉唑对 $\mathrm{H}^{+} \mathrm{K}^{+}$-ATPase 质子泵的抑制机制

Scheme 1 Esomeprazole for $\mathrm{H}^{+} \mathrm{K}^{+}$-ATPase inhibition

化为较为稳定的阿焦烯(Ajoene, 18) 以及其它有机硫化 物(Scheme 2) ${ }^{[21]}$. 阿焦烯是大蒜油提取物中的主要成分, 结构中包含亚砜官能团、二硫键和多烯烃双键. 众多的 研究表明它具有非常广泛的生物活性. 例如, 它是一种 细菌群体感应抑制剂(Quorum sensing inhibitor, QSI), 对 金黄色葡萄球菌和铜绿假单胞菌的生物膜形成具有抑 制活性 ${ }^{[22]}$, 进而展现出抗菌、抗炎作用. 另外, 阿焦烯 能够通过抑制血小板聚集来减少血栓的形成, 很大程度 上能降低人们罹患心脏病和中风等疾病的风险 ${ }^{21]}$.

2018 年, 英国卡迪夫大学化学系 Wirth 课题组 ${ }^{[23-24]}$ 运用化学合成手段实现了阿焦烯 18 的规模化制备 (Scheme 3, 约 $169 \mathrm{~g}$ ), 这一工作对进一步研究和评估阿 焦烯的药物活性有着重要意义. 与传统的大蒜素仿生热 重排制备方法 ${ }^{[21]}$ 相对比, 该方法具有更高的普适性, 也 为后续开展对阿焦烯的结构修饰或改造提供了一条非 常有效的途径 ${ }^{25-26]}$.

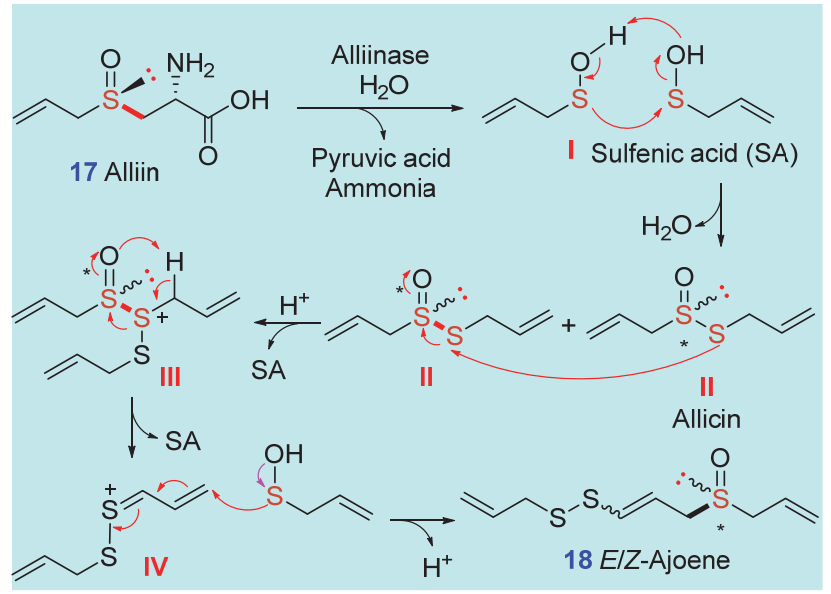

图式 2 阿焦烯的生物合成路线

Scheme 2 Biosynthetic route to ajoene

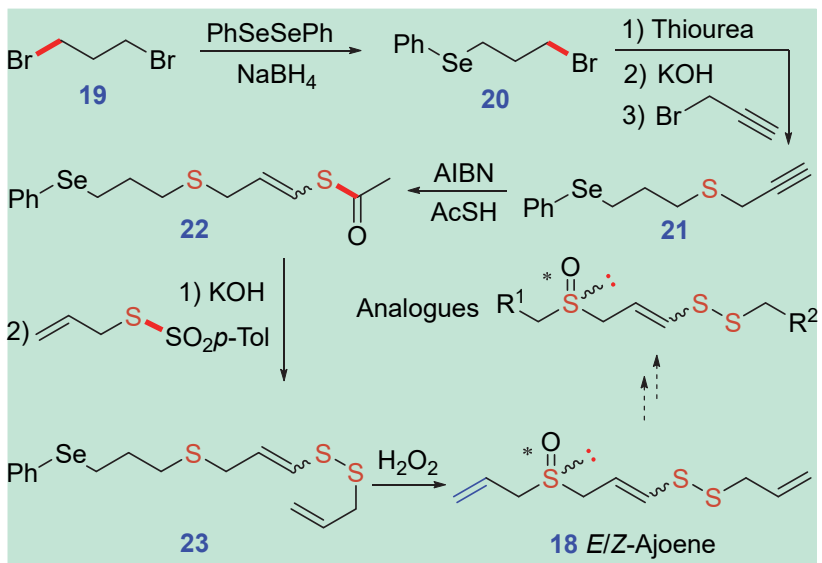

图式 3 阿焦烯以及其衍生物的化学合成路线

Scheme 3 Synthetic route to ajoene and its analogues

\section{3 萝卜硫素和乙烯基亚砜类化合物}

\subsection{1 萝卜硫素(Sulforaphane)}

萝卜硫素(Sulforaphane, 26)又称莱菔子素, 其化学 名为 1-异硫氰酸-4-甲基亚磺酰基丁烷(Scheme 4a), 包 含亚磺酰官能团和异硫氰酸基, 广泛存在于在西兰花、 花椰菜、甘蓝、北方圆红萝卜等十字花科植物中. 当植 物组织被破碎之后, 位于细胞质中的黑芥子酶能够接触 并催化水解原本存在于液泡中的硫苷(Glucoraphanin, 25), 水解产物再经由 Lossen 重排反应得到萝卜硫

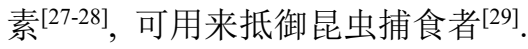

在动物模型的研究表明, 萝卜硫素能够通过活化具 有氧化还原敏感性的转录因子 Nrf2 (Nuclear factor E2-related factor 2)诱导血红素氧化酶 HO-1 (Hemeoxygenase-1)基因的表达，保护正常细胞免受氧化应激的损 伤, 进而起到防御肿瘤发生的作用. 此外, 萝卜硫素也 可以通过其他多种作用机制, 发挥其抗炎、抗癌、神经 保护等作用, 并且具有口服安全性. 值得一提的是, 萝 
(a)<smiles>CS(=O)(=O)CCCCN=C=S</smiles>

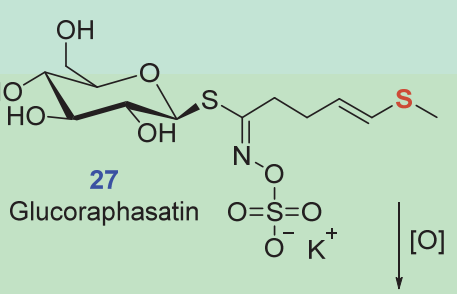

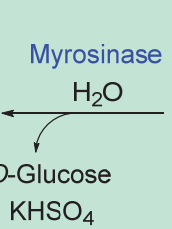

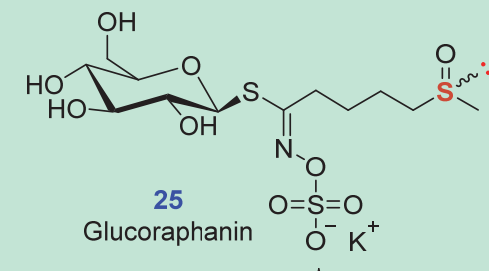

[O]
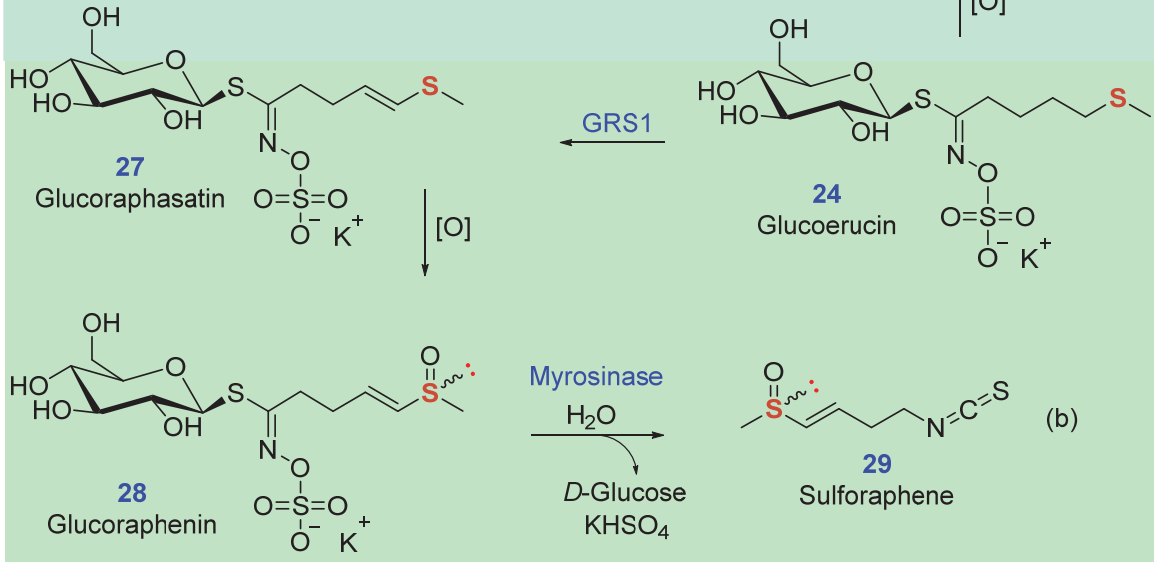

图式 4 萝卜硫素和莱菔素的生物合成路线

Scheme 4 Biosynthesis of Sulforaphane and Sulforaphene

卜硫素能够穿透血脑屏障, 并可以通过刺激胱硫醚 $\gamma$-裂 合酶 CSE (Cystathionine $\gamma$-lyase) 和胱硫醚 $\beta$-合酶 CBS (Cystathionine $\beta$-synthase)催化血管舒张剂 $\mathrm{H}_{2} \mathrm{~S}$ 的生成进 而增加脑血流量 ${ }^{[30]}$, 有望成为阿尔茨海默病等神经退 行性疾病的辅助治疗方法 [31].

\subsection{2 莱菔素}

萝卜籽(莱菔子)在中国的传统医学中已使用了一千 多年, 它所含的莱菔素 (Sulforaphene, 又称 Sativin 或 Raphanin, 29)具有很强的抗癌活性. 莱菔素与夢卜硫素 具有类似的结构, 两者的差别仅在于前者含有一个不饱 和双键，前者的结构为 $(E)$-4-异硫氰酸基-1-(甲基亚磺酰 基)-1-丁烯(Scheme 4b). 不饱和双键的产生, 归因于夢 卜中特有的编码 GRSI (Glucoraphasatin Synthase 1)蛋白 的基因 ${ }^{[32]}$. 通过 GRS1 酶催化 Glucoerucin 24 的脱氢反 应可得到 Glucoraphasatin 27, 之后再经由黑芥子酶催化 的水解反应获得莱菔素 29 (Scheme 4b).

Yang 课题组 ${ }^{[33]}$ 对莱菔素及其衍生物的抗癌活性进 行了系统研究. 他们发现, 莱菔素是染色体区域维持因 子 CRM1 (Chromosomal region maintenance 1)的小分子 拮抗剂, 而 CRM1 蛋白高表达与多种肿瘤的发生与发展 密切相关. 莱菔素含有异硫氰酸基团, 它可以通过共价 键结合, 靶向位于 CRM1 蛋白结合口袋中的半胱氨酸 Cys528 的巯基, 进而抑制 CRM1 蛋白的功能. 例如, 莱 菔素能够通过下调 CRM1 的表达, 诱导套细胞淋巴瘤发 生周期抑制和调亡, 进而发挥抗肿瘤的作用. 同样, 莱
菔素也能够抑制食道癌、人乳腺癌以及人肝癌 $\mathrm{HepG} 2$ 等癌细胞的增殖.

此外, Yang 课题组以莱菔素为起点, 通过理性结构 设计、计算机模拟对接篎选、药效学和药代动力学等实 验，发现其衍生物 $(E)$-4-异硫氰酸基-1-(3,5-二(三氟甲 基)苯基亚磺酰基)-1-丁烯(34, Scheme 5)对 CRM1 靶点 的抑制能力提高了近 30 倍. 其中, 3,5 -二(三氟甲基)苯基 官能团提供了额外的非共价作用力，提高了 34 与 CRM1 靶点的结合力, 34 作为 CRM1 的拮抗剂, 可以通 过调节炎症相关的 NF- $\mathrm{kB}$ 信号传导和 Nrf2 细胞防御途 径来逆转结肠炎, 在治疗结肠炎方面具有广阔的应用前 景 ${ }^{[34]}$.

值得一提的是, Liu 课题组 ${ }^{[35]}$ 以蜂巢蜂胶的主要活 性成分咖啡酸苯乙酯 35 为起点, 优化合成了 $(E)-3,4-二$ 羟基苯乙烯基苯丙基亚砜 36 (Scheme 6)，该结构展示了 抗氧化和抗神经炎症作用以及更高的血脑屏障通透性, 是一种潜在的多功能神经保护剂. 为进一步提高其药 效，并阐明构效关系，他们通过乙酰化修饰，合成了一 系列(E)-3,4-二乙酰氧基苯乙烯基苯丙基亚砜(如 37 38), 并通过帕金森氏病体外模型证明了其乙酰化结构 具有更优的抗神经炎症作用 ${ }^{[36]}$.

\subsection{Cenicriviroc 及其衍生物}

Cenicriviroc (39a, 图 3) 是一种靶向作用于 CCR2 和 CCR5 受体的双重拮抗剂[37], 日本武田 Takeda 公司已经 完成了其临床 II 期研究，主要考察其在治疗 HIV 病毒 


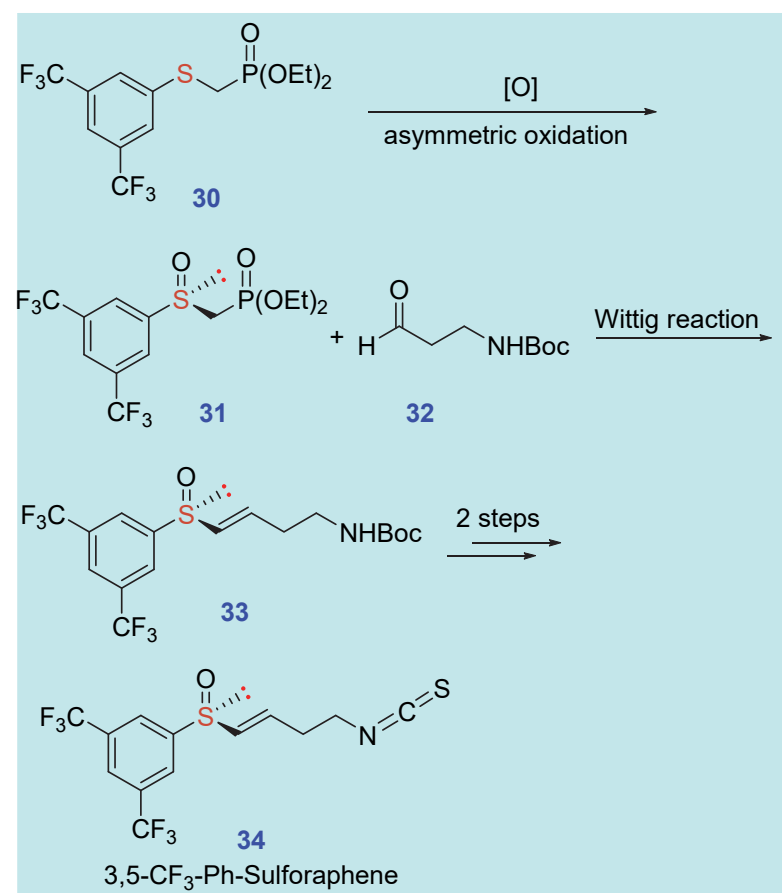

图式 5 莱菔素衍生物

Scheme 5 Sulforaphene derivative

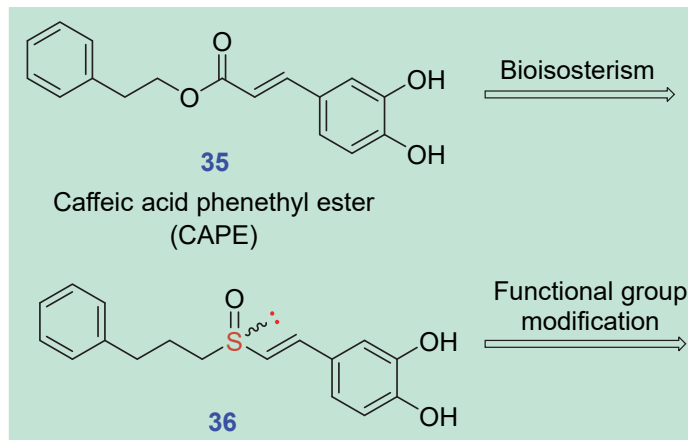

(E)-3,4-dihydroxystyryl aralkyl sulfoxide

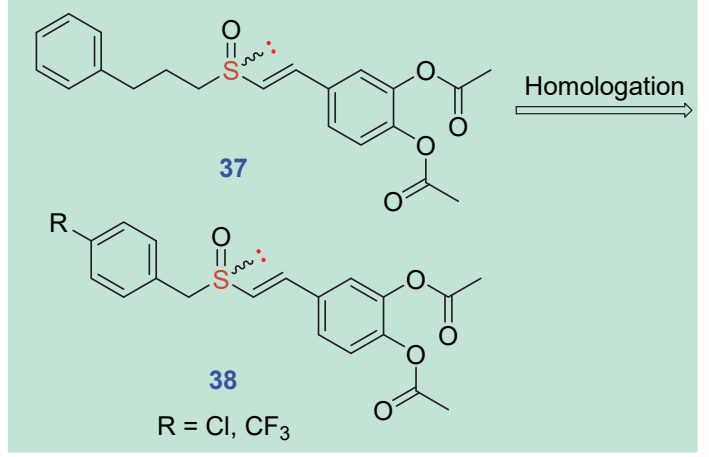

图式 6 乙烯基亚砜类化合物

Scheme 6 Vinyl sulfoxide compounds

感染以及非酒精性脂肪性肝炎等方面的应用. CCR2/ CCR5 均隶属于趋化因子受体 $(\mathrm{C}-\mathrm{C}$ chemokine receptor), 是表达在一些特定的细胞表面如白细胞表面的七 跨膜结构域蛋白, 包含一个 $\mathrm{N}$ 端细胞外区和一个 $\mathrm{C}$ 端细 胞质区，其中 $\mathrm{N}$ 端区域与趋化因子结合后， $\mathrm{C}$ 端区域与
$\mathrm{G}$ 蛋白偶联并在细胞内进行信号转导, 触发细胞内信号 级联反应. 因此, CCR 2 和 CCR 5 受体是药物研发中的一 类重要靶点, 其拮抗剂 Cenicriviroc 具有抗炎活性, 并能 够通过阻止 HIV 病毒进入人体宿主细胞进而展现抗 HIV 病毒活性且口服生物利用度高. 对 Cenicriviroc 进 行结构修饰，例如引入羧酸基团 ${ }^{[38]}$ 得到 39b(图 3), 通过 体外药效学和大鼠体内药代动力学研究, 发现具有良好 的药效、优秀的药代性质和显著的肝靶向性，可作为治 疗非酒精性脂肪肝病的候选药物。另外，打开 Cenicriviroc 结构骨架上的苯并氮杂环 ${ }^{[39]}$, 可以得到一系列 化合物如 40a $40 b$ (图 3), 对 CCR2 和 CCR5 受体同样 具有显著的拮抗作用.

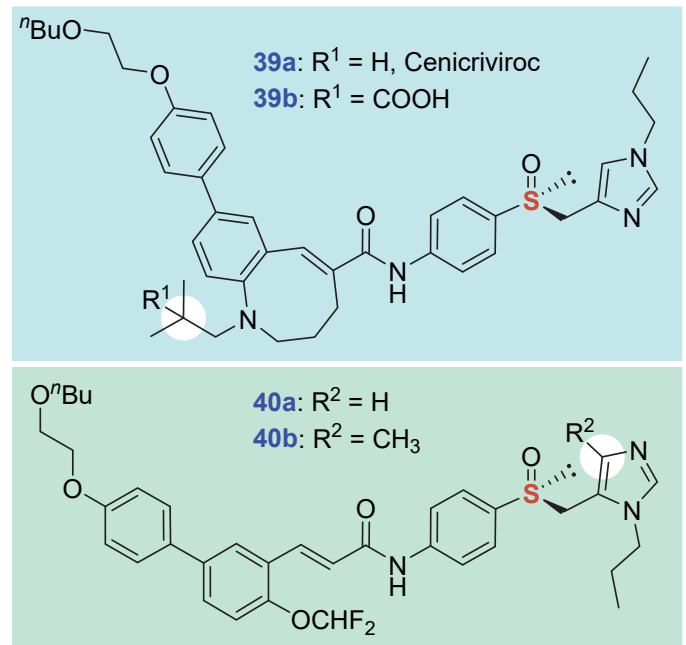

图 3 Cenicriviroc 及其衍生物

Figure 3 Cenicriviroc and its derivatives

\section{5 阿比多尔的亚砜衍生物}

2020 年 8 月, 国家卫健委颁布了《新型冠状病毒肺 炎诊疗方案(试行第八版)》，其中阿比多尔(Arbidol,

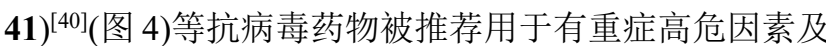
有重症倾向的患者的治疗. 阿比多尔是一种非核甘类广 谱抗病毒药物, 能够抑制病毒和人体宿主细胞膜的融 合，同时具有干扰素诱导和免疫调节的作用，可应用于 预防和治疗由轮状或冠状病毒引起的感染，尤其是肺 炎 ${ }^{[41]}$.

此外，研究表明，阿比多尔的亚砜衍生物 $\mathbf{4 2} \sim \mathbf{4 3}$ 能 够抑制乙肝病毒(Hepatitis B virus, HBV)的 DNA 复 制[42-43], 与阿比多尔相比，具有更低的细胞毒性和更显 著的抗 $\mathrm{HBV}$ 活性 $\left(\mathbf{4 2}: \mathrm{IC}_{50}=3.6 \mu \mathrm{g} / \mathrm{mL} ; \mathbf{4 3}: \mathrm{IC}_{50}=22.5\right.$ $\mu \mathrm{g} / \mathrm{mL}$ ). 同样, 亚砜 44 的抗丙肝病毒(Hepatitis C virus, $\mathrm{HCV}$ )的活性也得到了初步证实 ${ }^{[44]}$. 基孔肯雅病毒(Chikungunya virus, CHIKV)感染是一种以伊蚊为传播媒介 的急性传染病，临床上主要表现为发热、皮疹及关节疼 痛等症状. Filosa 课题组 ${ }^{[45-46]}$ 对阿比多尔进行了结构优 


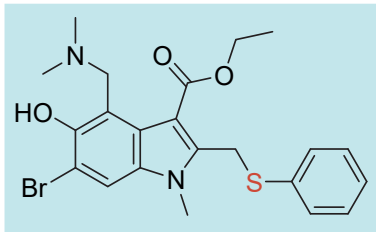

41

Umifenovir or Arbidol ${ }^{\circledR}$

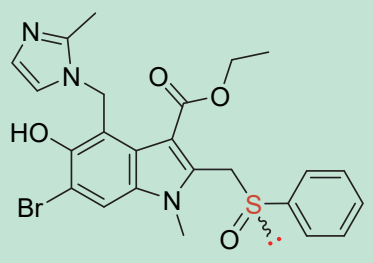

43 anti-HBV

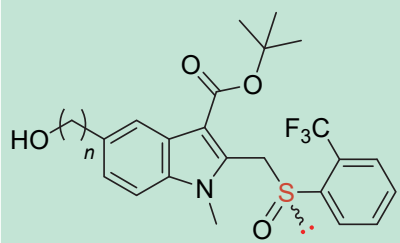

$n=0,1$

45 Inhibitor of CHIKV replication

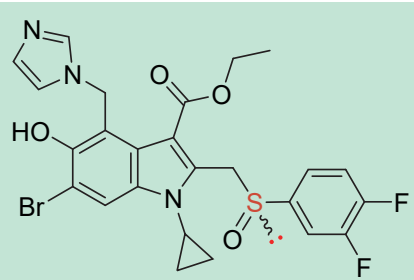

42 anti-HBV
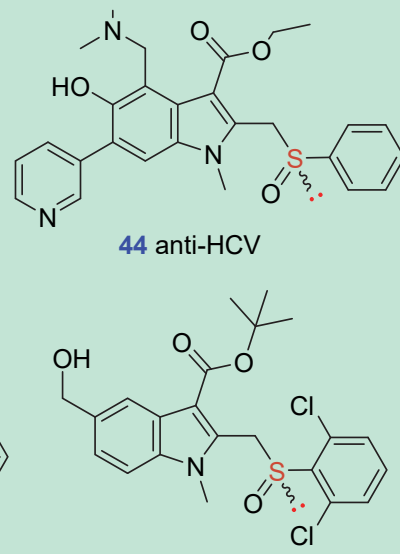

46 Inhibitor of CHIKV replication

图 4 阿比多尔及其亚砜衍生物

Figure 4 Arbidol and its sulfoxide analogues

化, 发现其亚砜衍生物 $\mathbf{4 5} \sim 46$ 具有良好的抗 CHIKV 活 性. 因此, 45 46 可作为苗头化合物应用于基孔肯雅热 病的药物开发进程之中.

\section{6 阿莫达菲尼}

阿莫达菲尼(Armodafinil，47a) 是一种唤醒促进剂 (图 5), 上市于 1998 年, 可用于治疗嗜睡症、轮班工作 睡眠障碍以及阻塞性睡眠呼吸暂停症等, 并且能够增强 认知和提高机敏性，但其确切的作用机制尚不清楚 ${ }^{[47]}$. 有研究表明, 它是一种非典型性多巴胺再摄取抑制剂 (Atypical dopamine reuptake inhibitor, ADRI), 药效靶点 可能是多巴胺转运蛋白(Dopamine transporter, DAT) ${ }^{[48]}$. 它可以通过抑制突触前神经元细胞膜上 DAT 的活性, 阻止存在于突触间隙的多巴胺被回收至突触前神经元 细胞, 从而增加突触后神经元细胞中多巴胺的浓度, 且 不会引起多巴胺的外溢, 无成瘾性和滥用倾向.

阿莫达菲尼及多巴胺两者与 DAT 的结合位点相互 重叠, 并且它们更倾向于与多巴胺转运蛋白的向内闭合 构象(Inward-facing conformation)结合 ${ }^{[48]}$, 而可卡因更倾 向结合多巴胺转运蛋白的向外开放构象(Outward-open conformation $)^{[49]}$. 综上所述, 阿莫达菲尼的药理学作用 机制明显区别于可卡因 DRI 等神经兴奋剂，可作为治疗 可卡因/甲基苯丙胺成瘾以及改善神经退行性疾病患者

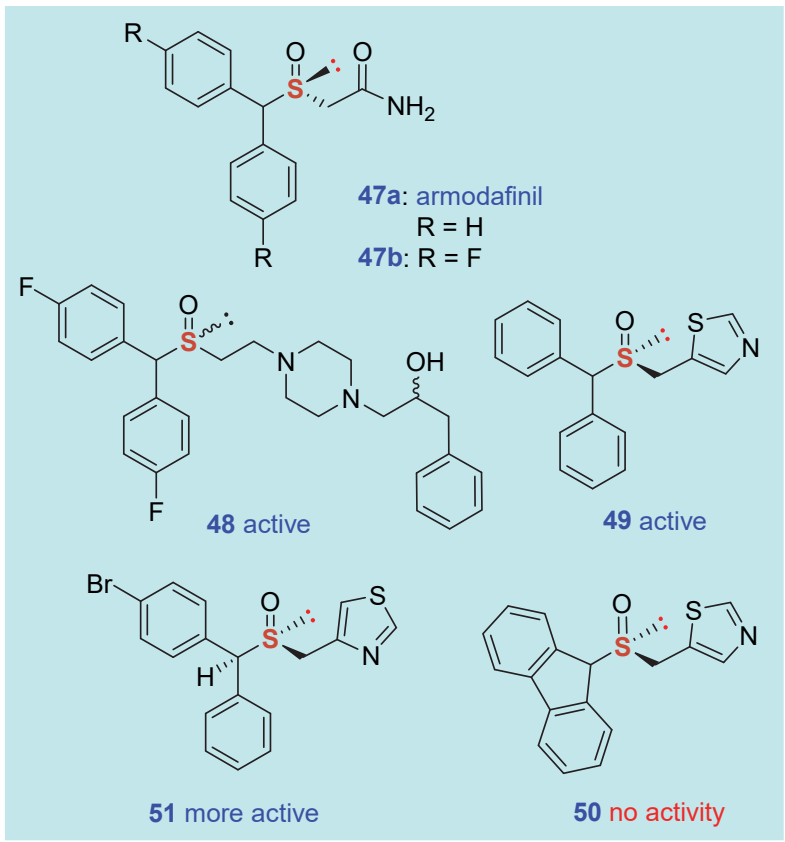

图 5 阿莫达菲尼及其衍生物

Figure 5 Armodafinil and its derivatives

认知能力的潜在药物.

为了进一步开发可应用于治疗精神兴奋剂滥用的 药物, 以阿莫达菲尼为先导化合物, Newma 课题组 ${ }^{[50]}$ 和 Lubec 课题组 ${ }^{[11-52]}$ 分别设计了其类似物 48 51 (图 5), 并 通过体外和大鼠体内研究提供了有关结构-活性关系的 宝贵见解. 其中含有两个手性中心的噻唑衍生物 51 对 DAT 具有亚微摩尔的抑制活性 $\left(\mathrm{IC}_{50}=0.65 \pm 0.07\right.$ $\left.\mu \mathrm{mol} \cdot \mathrm{L}^{-1}\right)$ 和较高的选择性, 它的抑制能力比阿莫达菲 尼高 10 倍以上. 此外, 阿莫达菲尼的衍生物 $47 \mathrm{~b}$ 能够通 过抑制钙激活钾离子通道 $\mathrm{K}_{\mathrm{Ca}} 2.3$ 蛋白表达进而抑制肝 纤维化和肺纤维化 ${ }^{[53]}$, 是预防和治疗肝病的潜在药物.

\section{7 其他亚砜类化合物的生物活性}

舒林酸(Sulindac, 52)(图 6)是一种非甾体类抗炎镇 痛解热药, 其确切的作用机理尚不清楚. 一般认为, 它 可以通过抑制环氧合酶 COX- $1 / 2$ 来干扰与炎症发生相 关的前列腺素的合成，从而发挥抗炎作用. $\mathbf{5 3}^{[54]}$ 是用来 制备手性亚砜亚胺 AZD6738 ${ }^{[55]}$ 的关键中间体，而 AZD6738 是一种具有口服活性的 ATR 激酶抑制剂, 可 阻止癌细胞的自我修复. 目前 AZD6738 已进入临床 I/II 试验，在癌症治疗领域具有潜在的应用前景. Oxisuran $\mathbf{5 4}^{[56-57]}$ 是一种免疫抑制剂, 在动物皮肤或器官移植试验 中能够发挥一定的积极作用.

含有亚磺酰基的噁二唑类化合物 55 (图 6) ${ }^{[58]}$ 是一种 新型糖原合酶激酶-3 3 (Glycogen Synthase Kinase-3 $\beta$, GSK-3 $\beta$ )抑制剂, $\mathrm{IC}_{50}$ 值分别为 34 和 $20 \mathrm{nmol} \cdot \mathrm{L}^{-1}$. 小鼠 试验表明, 它具有良好的血脑屏障通透性和药代动力学 


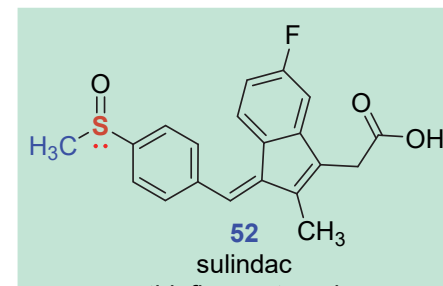

anti-inflammatory drug

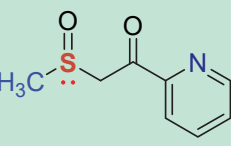

54

Oxisuran immunosuppressive agent

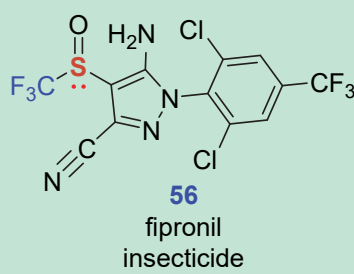

图 6 舒林酸和其他具有生物活性的亚砜

Figure 6 Sulindac and other bioactive sulfoxides

特征, 能够抑制与阿尔兹海默病相关的 Tau 蛋白的过度 磷酸化. Fipronil 56 $\mathbf{6}^{[59]}$ 能够通过阻断 $\gamma$-氨基丁酸以及谷 氨酸门控氯离子通道来扰乱昆虫的中枢神经系统, 是一 种广谱杀虫剂. 含有甲基葱和吡啶基的亚砜 ZD3638 (57) $)^{[60-61]}$ 是由阿斯利康开发的一种非经典抗精神病候选 药物, 对多巴胺 $\mathrm{D}_{1} / \mathrm{D}_{2}$ 受体和血清素 $5-\mathrm{HT}_{2}$ 受体均具有 拮抗作用, 在治疗精神分裂症等精神疾病中具有潜在价 值.

幽门螺杆菌(Helicobacter pylori, Hp)感染是诱发消 化性溃疡和胃癌疾病的主要原因. 化合物 58(图 7) ${ }^{[62]}$ 可 作为幽门螺杆菌黄酮毒素氧化还原蛋白的抑制剂, 并具 有较高的治疗指数(therapeutic index)和很低的小鼠口服 毒性, 有助于对抗持续增长的耐抗生素幽门螺杆菌株. RP 73163 (59) ${ }^{[63-65]}$ 是一种特异性的酰基辅酶 A:胆固醇 酰基转移酶(Acyl-CoA:cholesterol acyltransferase, ACAT) 抑制剂, 能够降低 ACAT 的活性和极低密度脂蛋白中胆 固醇酯的含量, 并减缓了极低密度脂蛋白的分泌, 通过 降低血浆中胆固醇的浓度来发挥降血脂作用。 OPC$29030(\mathbf{6 0})^{[66-67]}$ 能够通过阻止血小板中的 12-酯氧合酶 移位至细胞膜来抑制 12-羟基二十碳四烯酸(12-Hydroxyeicosatetraenoic acid, 12-HETE)的产生, 而 12-HETE 与粘附糖蛋白以及 $\mathrm{P}-$ 选择素蛋白的表达息息相关, 因此 OPC-29030 (60)具有抑制血小板粘附的作用, 在治疗和 预防血栓形成方面具有一定的应用价值.

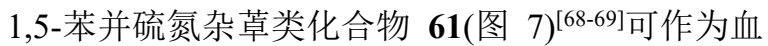

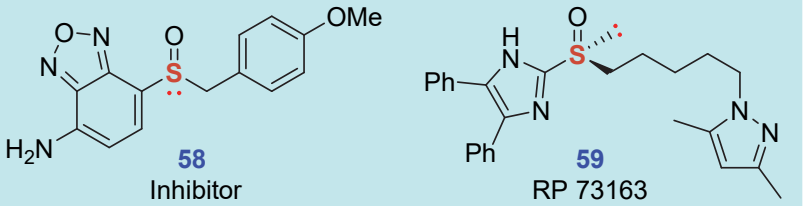

of Flavodoxin

with hypolipidemic effect

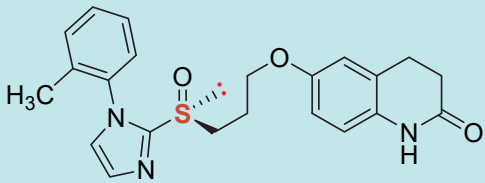

60

OPC-29030

platelet adhesion inhibitor

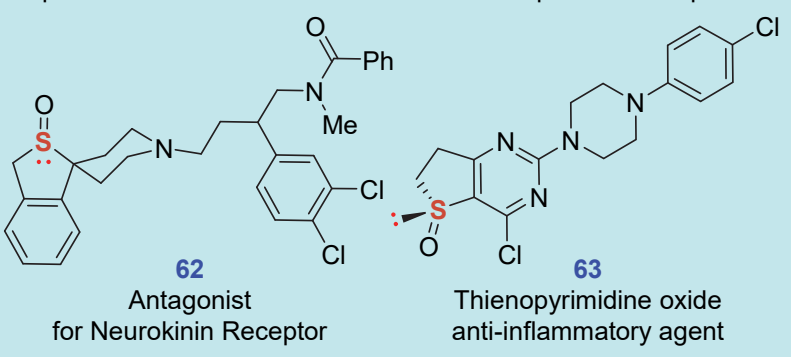

图 7 具有生物活性的杂芳基亚砜和环亚砜

Figure 7 Bioactive heteroaromatic and cyclic sulfoxides

管加压素受体的配体，有利于清除体液潴留，对治疗低 钠血症以及充血性心力衰竭水肿等疾病有着潜在的应 用价值. 螺环化合物 YM-38336 (62) ${ }^{[70]}$ 是神经激肽-2 (Neurokinin-2, NK2)受体的拮抗剂, 在治疗与 NK2 受体 活化相关的疾病中有一定的临床应用价值, 如在豚鼠研 究模型中, 62 能抑制支气管收缩和十二指肠痉挛。二氢 噻吩并嘧啶类化合物 $\mathbf{6 3}^{[71]}$ 是勃林格殷格翰公司正在研 发的一种抗炎药物，适用于治疗呼吸道或胃肠道不适以 及关节、皮肤或眼睛的炎症性疾病 ${ }^{[72]}$.

\section{2 亚砜类化合物的不对称合成}

随着手性亚砜化合物在各领域发挥的作用越来越 重要, 通过各种不对称催化的策略来合成亚砜也成为越 来越热门的话题. 本文接下来将重点阐述近十年来有关 于亚砜的不对称催化合成的方法，同时也列举了能够成 功合成前文所提到的埃索美拉唑 $(10)$ 以及阿莫达菲尼 (47a)等手性亚砜药物的路线.

有关手性亚砜化合物的制备可追溯到 1926 年, Phillips 等[73]采用 $(+)$-樟脑磺酸作为手性拆分剂经由化 学拆分法得到光学活性的亚砜. 1962 年 Andersen ${ }^{[74]}$ 发展 了手性醇助剂诱导法, 以重结晶分离得到的光学纯亚磺 酸-(一)-薄荷酯 (64a)为原料(Scheme 7a), 与格氏试剂或 有机酮锂试剂发生取代反应，通过 $\mathrm{S}-\mathrm{C}$ 键的构建得到 具有高对映过量值的手性亚砜. 1984 年, Kagan 课题 组 ${ }^{[75-76]}$ 和 Modena 课题组 ${ }^{[77]}$ 分别对 Sharpless 环氧化 $\mathrm{Ti}\left(\mathrm{O}^{i} \mathrm{Pr}\right)_{4} /(R, R)-$ 酒石酸乙酯 (70) 催化体系进行改良 
(Scheme 7b), 使之适用于硫醚不对称氧化反应, 为手性 亚砜的大规模制备提供了技术支撑，同时开启了手性金 属络合物(如 $\mathrm{V}, \mathrm{Mn}, \mathrm{Fe}, \mathrm{Cu}, \mathrm{Zr}, \mathrm{Mo}, \mathrm{W}, \mathrm{Os}, \mathrm{Al}$ 等)催化氧 化体系的蓬勃发展. 相关的研究情况可参考系统的综述 文献[78-81]，因篇幅有限，在此就不一一列举.

a) Anderson method via diastereochemically pure sulfinates 64

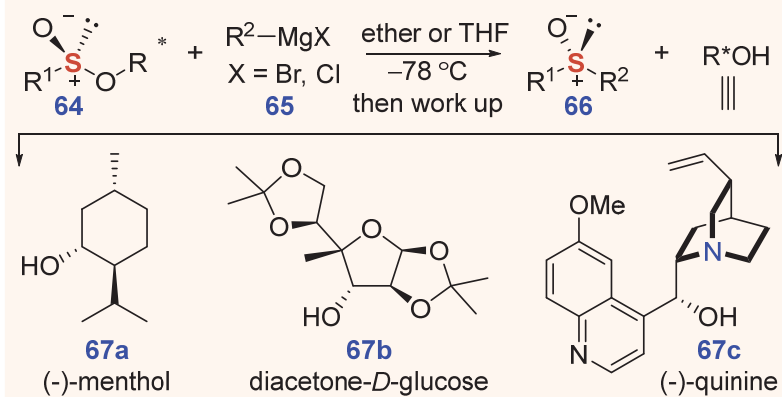

b) Kagan and Moderna methods of asymmetric sulfoxidation

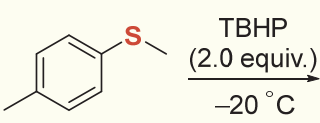

68

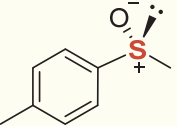

69

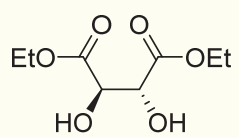

$70(R, R)-\mathrm{DET}$
Kagan: $\mathrm{Ti}\left(\mathrm{O}^{\prime} \mathrm{Pr}\right)_{4}:(R, R)-\mathrm{DET}: \mathrm{H}_{2} \mathrm{O}=1: 2: 1, \mathrm{CH}_{2} \mathrm{Cl}_{2}, 93 \%$ ee Modena: $\mathrm{Ti}\left(\mathrm{O}^{j} \mathrm{Pr}\right)_{4}:(R, R)-\mathrm{DET}=1: 4, \mathrm{CHCl}_{2} \mathrm{CHCl}_{2}, 88 \%$ ee

图式 7 Anderson 法和 Kagan-Modern 法制备手性亚砜

Scheme 7 Anderson method and Kagan-Moderna method

经过将近一百年的探索, 手性亚砜合成领域已经取 得了显著进展(图 8), 从需要化学计量的手性拆分剂或 手性助剂, 到只需催化计量的手性催化剂, 以及涌现的 新催化剂体系和合成新策略. 本文将着重介绍近十年有 关手性亚砜合成的重要进展和新策略 ${ }^{[81]}$, 包括廉价金 属铁络合物催化剂、多金属氧酸盐、有机小分子催化剂、 生物催化和电催化作用下的硫醚不对称氧化反应, 以及 经由次磺酸阴离子中间体的转化途径等策略.

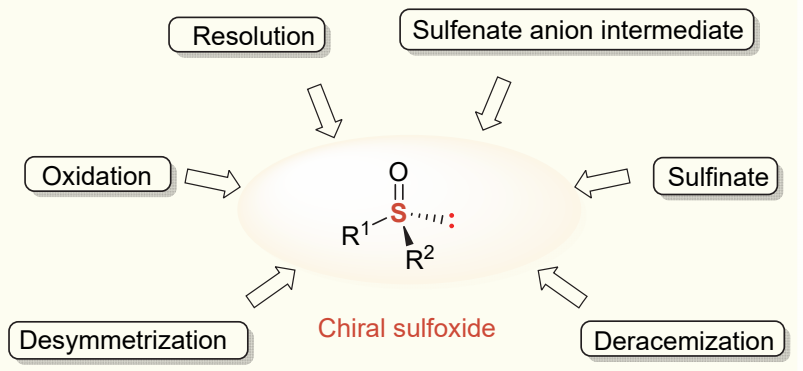

图 8 手性亚砜的合成路径

Figure 8 Synthetic approaches to chiral sulfoxides

\section{1 铁络合物催化氧化体系}

与其他过渡金属相比，采用价廉、丰富且低毒的铁 盐作为催化剂[82], 并以 $\mathrm{H}_{2} \mathrm{O}_{2}$ 为氧化剂, 通过硫醚不对
称氧化反应合成手性亚砜，是一种 “可持续” 的催化体 系.

已有报道表明，铁盐与席夫碱 $\mathbf{7 4}^{[83]} 、 N, N^{\prime}$-二氮氧配 体 $\mathbf{7 5}^{[84]}$ 、Salan 76 ${ }^{[85]}$ 、卟啉 $7^{[86]}$ 等手性配体络合而成的 催化剂 [79]，展示了良好的催化活性(Scheme 8). 例如, Feng 课题组 ${ }^{[84]}$ 以 $\mathrm{Fe}(\mathrm{OTf})_{3}$ 与 $N, N^{\prime}$-二氮氧配体 75 形成的 络合物作为路易斯酸催化剂, 通过活化 $\mathrm{H}_{2} \mathrm{O}_{2}$, 可催化一 系列硫醚底物的不对称氧化反应(Scheme $8 b$ ), 且官能 才兼容性好，产物对映选择性高. 结合电子顺磁共振谱 和紫外-可见光谱方法，作者探讨了可能的反应机理， 初步推测高自旋 $\mathrm{Fe}^{\mathrm{III}}-\mathrm{OOH}$ 配合物可能是该体系中真正 的氧化催化活性物质, 且实现了阿莫达菲尼 47a 的克级 规模制备. 阿莫达菲尼作的药理作用在前文介绍手性亚 砜类生物活性时已有提及，此处不再赘述.

\section{2 多金属氧酸盐催化氧化体系}

除了有机配体一过渡金属络合物催化剂之外，多金 属氧酸盐(Polyoxometalates, POMs)也是一种优良的氧 化催化剂. 多金属氧酸盐阴离子一般由多原子 $(\mathrm{V}, \mathrm{Nb}$, $\mathrm{Ta}, \mathrm{Cr}, \mathrm{Mo}, \mathrm{W}$ 等)和杂原子( $\mathrm{Si}, \mathrm{P}, \mathrm{S}$ 等)按照一定的空间 排布通过氧原子配位桥联而成, 其结构具有多样性、可 修饰性以及可调控性, 可应用于烯烃环氧化 ${ }^{[87-88]}$ 和硫醚 氧化 ${ }^{[89-92]}$ 等反应.

理论上，多金属氧酸盐阴离子与手性抗衡阳离子形 成的手性离子对催化剂, 可以通过活化氧气或双氧水来 实现不对称催化氧化反应. 然而, 其挑战之一就是如何 通过静电引力而不是配位键来控制反应的立体选择性. 2011 年, Nlate 团队 ${ }^{[91-92]}$ 将磷钨杂多酸 $\mathrm{H}_{3} \mathrm{PW}_{12} \mathrm{O}_{40}$ 与基于 (S)-1-苯乙胺的树枝状手性三胺反应, 得到了手性磷铇 杂多酸三聚铵 78 (Scheme 9a). 该催化剂在苯基甲基硫 醚氧化反应中的对映选择性为 $13 \% \mathrm{ee}$, 作者通过一系 列的结构表征不排除手性三胺作为铇配体的可能. 2016 年, Tan 课题组 ${ }^{[93-94]}$ 制备和解析了含氧桥硫酸根过氧化 双核钼酸-手性双胍离子对催化剂 79 的晶体结构 (Scheme 9b) $)^{[87]}$, 确定了手性双胍作为抗衡阳离子在硫 醚不对称氧化反应中的高催化活性和高对映选择性. 通 过反应条件的篎选和优化, 分别采用钼酸钠和铇酸银作 为金属氧酸盐, 在 $\mathrm{H}_{2} \mathrm{O}_{2}$ 条件下实现了阿莫达菲尼 47a 和 $(S)$-兰索拉唑 13 的手性合成(Schemes $9 \mathrm{c} \sim 9 \mathrm{~d})$. 该催 化体系采用低投量的手性抗衡阳离子代替手性配体，为 手性过渡金属催化体系提供了新思路，反应条件温和， 有望应有于手性亚砜类药物的工业生产.

\section{3 有机小分子催化氧化体系}

和 Shi 手性酮催化剂[95]和 $\beta$-环糊精-黄素手性催化 剂[96]一样，手性磷酸有机小分子催化剂也可以催化硫 
a)
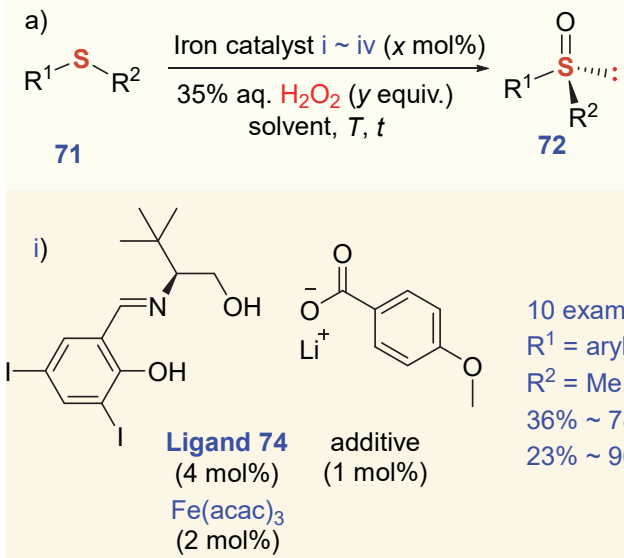

$x=2$, solvent: $\mathrm{CH}_{2} \mathrm{Cl}_{2}, y=1.2, T=20^{\circ} \mathrm{C}, t=16 \mathrm{~h}$

iii)

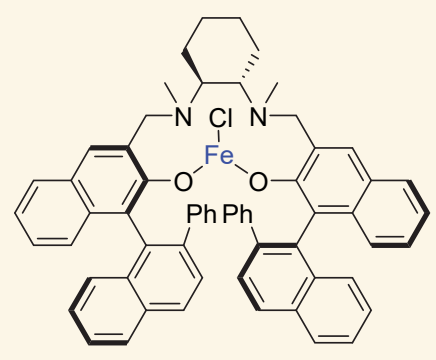

Cat. 76
12 examples

$\mathrm{R}^{1}=$ aryl, alkyl

$\mathrm{R}^{2}=\mathrm{Me}, \mathrm{Et}$

$76 \% \sim 99 \%$ yield

$81 \% \sim 96 \%$ ee<smiles>NC(=O)CSC(c1ccccc1)c1ccccc1</smiles><smiles>[Te]=[Te]</smiles><smiles>C[C@](CC(N)=O)(c1ccccc1)C([O-])c1ccccc1</smiles>

ent-75b, MTBE, $35{ }^{\circ} \mathrm{C}, 1.004 \mathrm{~g}, 92 \%$ yield, $87 \%$ ee

ii)

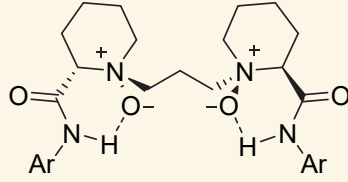

31 examples

$\mathrm{R}^{1}=$ aryl, alkyl;

$\mathrm{R}^{2}=$ alkyl, benzyl, allyl, amide

Ligand 75 (12 mol\%)

$\mathrm{Fe}(\mathrm{OTf})_{3}(10 \mathrm{~mol} \%)$

$36 \% \sim 78 \%$ yield

$23 \% \sim 96 \%$ ee

75a: $\mathrm{Ar}=2,6-{ }^{i} \mathrm{Pr}_{2}-4-\left(1\right.$-adamantyl) $\mathrm{C}_{6} \mathrm{H}_{2}$

$75 \mathrm{~b}: \mathrm{Ar}=2,6-\mathrm{Et}_{2}-4-\mathrm{MeC}_{6} \mathrm{H}_{2}$

$x=10$, solvent: THF, $y=6, T=25^{\circ} \mathrm{C}, t=4 \sim 48 \mathrm{~h}$

iv)

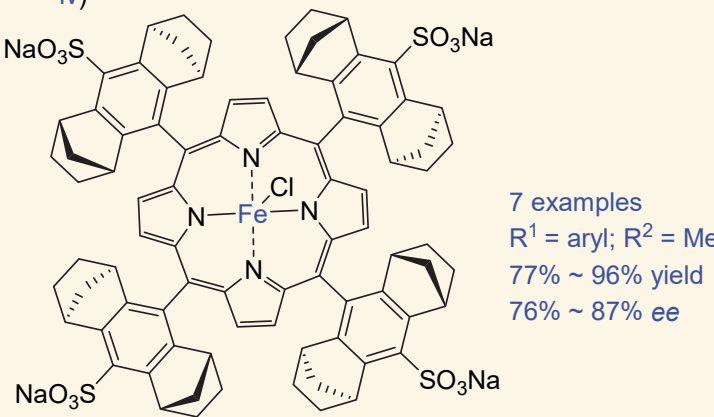

Cat. 77

$x=1$, solvent: andryous $\mathrm{MeOH}, y=1.2, T=-20^{\circ} \mathrm{C}, t=1 \mathrm{~h}$

图式 8 铁络合物催化的硫醚不对称氧化反应

Scheme 8 Iron-catalyzed asymmetric sulfoxidation

醚不对称氧化反应. 手性磷酸是一类具有较强酸性的 Brønsted 酸催化剂，且分子内同时含有 Brønsted 酸性位 点(磷原子上的羟基氢)和 Lewis 碱性位点(磷原子上的双 键氧), 是一种新型双功能有机催化剂. 其可能的催化 机理如下: 手性磷酸通过双氢键作用活化 $\mathrm{H}_{2} \mathrm{O}_{2}$, 同时利 用位阻效应有效地控制硫醚底物的进攻方向, 从而取得 高对映选择性(Scheme 10). 例如, 2012 年 Wang 课题 组 ${ }^{[97]}$ 和 List 课题组 ${ }^{[98]}$ 分别采用手性磷酸 84 和手性酰亚 胺基二磷酸 85 , 实现了对芳基/烯基/烷基-烷基手性亚 砜以及 $(R)$-舒林酸 $(\mathbf{5 2})$ 的高效合成, 尤其是在手性磷酸 85 催化体系下, 获得的对映选择性不逊于金属催化体 系给出的结果.

目前，除了基于手性酮、黄素手性衍生物以及手性 磷酸, 能够适用于硫醚不对称氧化的有机小分子催化剂 寥翏无几. 2020 年, Tang 课题组 ${ }^{[99}{ }^{3}$ 参考黄素的氧化催化 活性中间体氢过氧黄素(Scheme 10), 设计了一种结构 新颖的手性四氧嘧啶催化剂 86, 将其应用在硫醚不对 称氧化反应中. 尽管反应只能得给出中等的对映选择性 $(60 \% e e)$, 但为进一步开发高效的有机小分子催化剂提 供了新思路.

\section{4 生物催化氧化体系}

近年来，随着基因组学、生物信息学、合成生物学、 酶定向演化等研究领域的突飞猛进，生物催化技术获得 了巨大进展，人们不但可以发现酶、认识酶、改造酶，甚 至可以创造酶，进而提高酶的催化活性、选择性和底物 范围，促进绿色、高效的手性化学品制造体系的形成. 在生物催化的硫醚不对称氧化反应体系中, 酶催化剂的 主要来源包括微生物、纯酶和蛋白质工程酶 ${ }^{[100]}$. 例如, $\mathrm{Xu}$ 课题组通过域聚焦诱变和随机诱变等蛋白质工程方 法, 得到具有亮氨酸和脯氨酸两个突变位点的环己酮单 加氧酶 $A c \mathrm{CHMO}_{\mathrm{M} 7}(\mathrm{~L} 55 \mathrm{Y} / \mathrm{P} 497 \mathrm{~S})$, 在提高酶催化效率 的同时，减弱奥美拉唑硫醚底物 88 对酶的抑制作用，底 物浓度可达 $15 \mathrm{mmol} \cdot \mathrm{L}^{-1}$, 在 $6 \mathrm{~h}$ 内以 $97 \%$ 的产率获得 光学纯 $(S)$-奥美拉唑 $(\mathbf{1 0})^{[101]}$. (S)-奥美拉唑具有很好的抑 制胃酸分泌和治疗胃溃疡的作用，其抑制机制可参考前 文(Scheme 1). AcCHMO 催化反应体系具有原子经济性 高、环境友好等特点，以 $57.8 \%$ 的收率实现了埃索美拉 唑(10)的中试生产 (Scheme 11) ${ }^{[102]}$, 其中辅酶 NADPH 可 经由甲酸钠脱氢反应得以再生, 氧化剂为 $\mathrm{O}_{2}$, 副产物仅 为 $\mathrm{CO}_{2}$ 和 $\mathrm{H}_{2} \mathrm{O}$, 整体上展现了良好的绿色工业化前景. 
a)

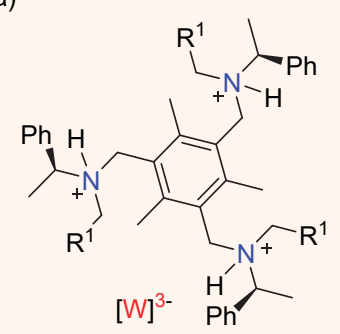

$\mathrm{R}^{1}=4-\mathrm{C}\left({ }^{n} \mathrm{Pr}\right)_{2} \mathrm{OH}-\mathrm{C}_{6} \mathrm{H}_{4}$

$(S, S)-78-[\mathrm{W}]$

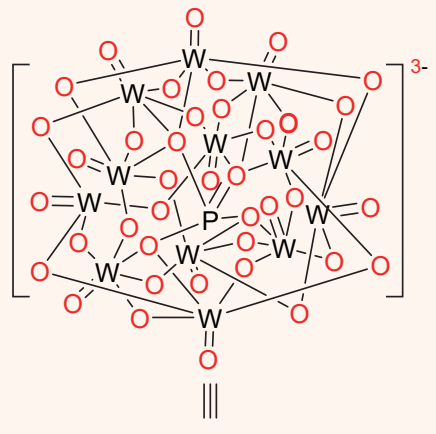

$[\mathrm{W}]^{3-}$ b)

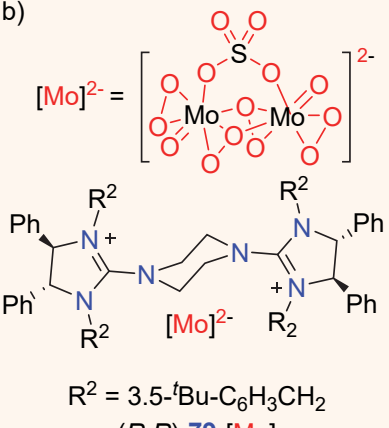

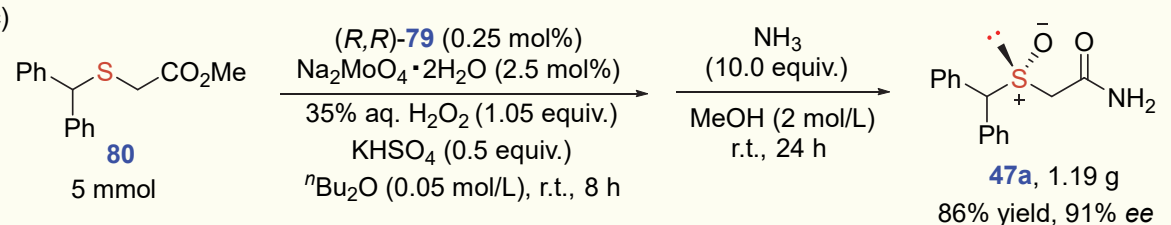

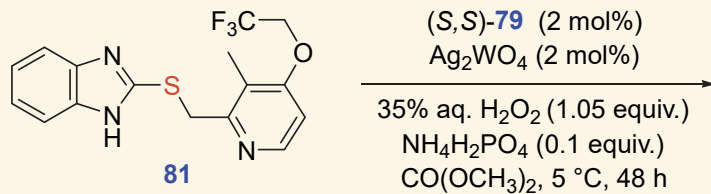

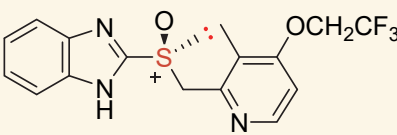

(S)-13 81\% yield, $90 \%$ ee

图式 9 多金属氧酸盐催化的硫醚不对称氧化反应

Scheme 9 Asymmetric sulfoxidation catalyzed by polyoxometalates

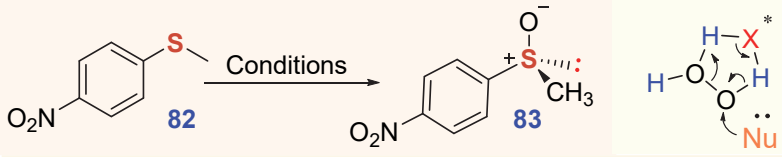

Conditions

a) cat. 84 (10 mol\%), $50 \mathrm{wt} \% \mathrm{H}_{2} \mathrm{O}_{2}$ (1.50 equiv.), $\mathrm{CHCl}_{3},-40{ }^{\circ} \mathrm{C}$, $144 \mathrm{~h}, 42 \%$ yield, $66 \%$ ee, 83

b) cat. 85 (2 mol\%), $35 \mathrm{wt} \% \mathrm{H}_{2} \mathrm{O}_{2}$ (1.05 equiv.), $\mathrm{MgSO}_{4}$, cyclohexane, r.t., $2 \mathrm{~h}, 95 \%$ yield, $99 \%$ ee, ena- 83

c) cat. $86(10 \mathrm{~mol} \%), 30 \mathrm{wt} \% \mathrm{H}_{2} \mathrm{O}_{2}$ (1.20 equiv.), $\mathrm{MgSO}_{4}, \mathrm{CF}_{3} \mathrm{CH}_{2-}$ $\mathrm{OH},-30{ }^{\circ} \mathrm{C}, 24 \mathrm{~h}, 92 \%$ yield, $60 \%$ ee, ena- 83

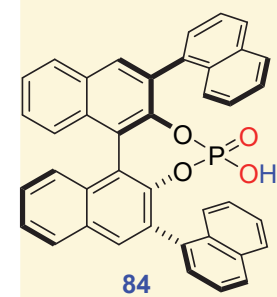

Phosphoric acid

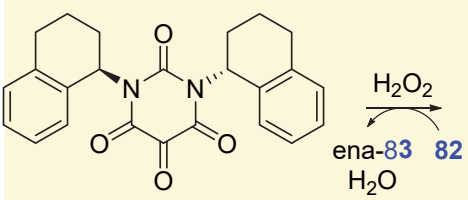

86

Alloxan

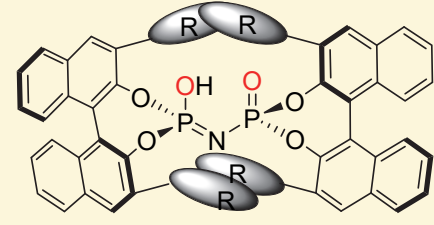

$\mathrm{R}=2,4,6-\mathrm{Et}_{3} \mathrm{C}_{6} \mathrm{H}_{2}$

85 Imidodiphosphoric acid

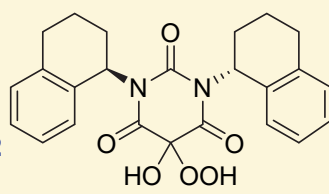

87

Alloxan hydroperoxide
图式 10 有机小分子催化的硫醚不对称氧化反应

Scheme 10 Organocatalyst for asymmetric sulfoxidation

\section{5 电化学催化氧化体系}

运用电化学技术, 快速、清洁、温和地合成手性化 合物，是一个值得关注的研究领域，目前正处于快速发 展阶段 ${ }^{[103-105]}$. 早在 1976 年, Miller 课题组 ${ }^{[106]}$ 就研究了 共价键型手性修饰电极石墨电极在不对称硫醚氧化反 应中的应用(Scheme 12a). 其电极修饰过程如下：石墨 电极在高温氧化条件下发生羧基化, 再经由酰氯化, 与 (S)-苯丙胺甲酯反应生成酰胺(Scheme 12b). 将该手性 修饰电极用于对甲基-苯基甲基硫醚的氧化反应中，亚 砜 90a 的产率高达 90\%, 但是对映过量值仅为 $2.5 \%$.

为提高电极表面手性物质覆盖率和稳定性，1984 年, Nonaka 课题组 ${ }^{[107]}$ 将聚吡咯薄膜共价键修饰的铂电 极浸入到聚 $L$-烦氨酸的三氟乙酸溶液中, 然后减压抽干 得到了聚吡咯和聚 $L$-缬氨酸双层薄膜修饰的铂电极 (Scheme 12c). 采用此类电极对一系列芳基烷基硫醚进 行不对称氧化, 其中亚砜 90b 产物的对映过量值高达 93\%, 化学收率为 $45 \%$. 随着电化学合成技术和相关理 论的不断进步和完善, 借助于电能而非化学氧化剂的不 对称硫醚氧化反应正在迎来新的发展契机.

\section{6 次磺酸阴离子策略}

在生物体系中，含颈基蛋白质部分氧化而成的次磺 酸化蛋白, 是生物体感受氧化应激的重要瞬时标志物. 


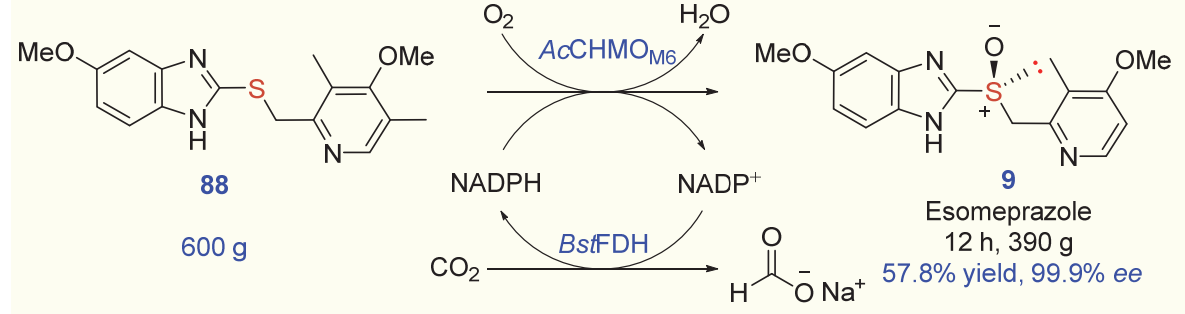

$\mathrm{AcCHMO}_{\mathrm{M} 6}$ : Acinetobacter calcoaceticus Cyclohexanone Monooxygenase mutation variant 6 BstFDH: Burkholderia stabilis 15516 Formate Dehydrogenase

图式 11 生物催化氧化法应用于中试生产 $(S)$-奥美拉唑

Scheme 11 Pilot-scale biocatalytic sulfoxidation for $(S)$-omerprazole

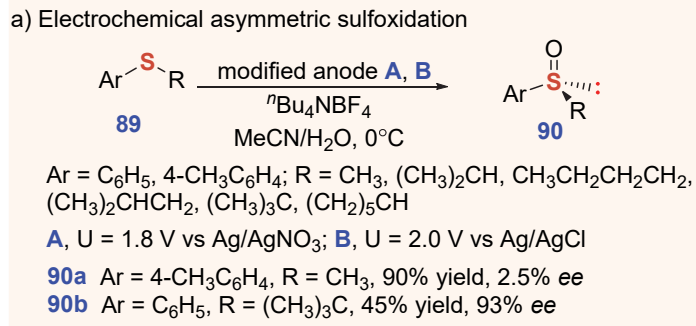

b) Covalently modified graphite anode

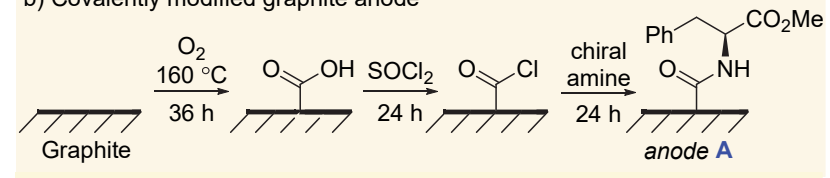

c) Polypyrrole- and poly(L-valine)-coated Pt anode
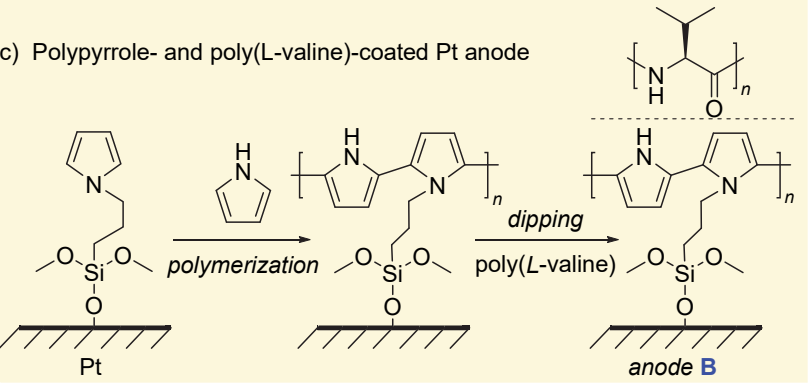

图式 12 电化学法硫醚不对称氧化反应

Scheme 12 Electrochemical asymmetric sulfoxidation

在化学体系中, 原位生成的次磺酸阴离子与亲电试剂发 生反应形成 $\mathrm{S}-\mathrm{C}$ 键, 可作为构建亚砜化合物的新策略. 早在 2004 年, O'Donnell 和 Schwan ${ }^{[108]}$ 就阐述了次磺酸阴 离子的结构、制备方法以及反应活性. Scheme 13 介绍了 几种可用于原位制备次磺酸阴离子中间体的前体化合

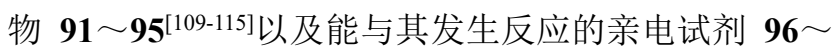
$9^{[116-118]}$.

2005 年, Perrio 课题组 ${ }^{[109]}$ 以外消旋的 $\beta$-对甲苯亚磺 酰基-丙酸甲酯为前体, 采用 $n$ - BuLi 以及手性配体(一)鹰爪豆碱 100, 通过碱促进的逆 Michael 加成反应原位 生成次磺酸阴离子, 经由与碘甲烷的取代反应, 获得了 具有 $29 \%$ ee 值的苯基甲基亚砜. 在 2007 年, Poli 和 Madec 课题组 ${ }^{[110]}$ 采用三(二亚苄基丙酮)二钯 $\left[\mathrm{Pd}_{2}(\mathrm{dba})_{3}\right]$
与 Josiphos 配体 101 催化剂体系, 在碱性条件下, 实现 了对次磺酸阴离子的不对称芳基化反应，得到了具有较 高对映体过量的亚砜 103 (80\% ee) (Schemes 14, i).

在此基础上, 2018 年, Zhang 课题组 ${ }^{[119]}$ 采用一种新 型的手性亚砜酰胺膦配体 $\mathbf{1 0 2}$ (Scheme 14a, ii), 极大地 扩宽了 $\mathrm{Pd}_{2}(\mathrm{dba})_{3}$ 催化体系的底物适用范围, 合成了包括 $(R)$-舒林酸 52 在内的 116 个手性亚砜化合物，且大部分 都具有高对映体过量 $(86 \%$ \% $99 \% e e)$ ，进一步验证了该 策略的实用性. Walsh 课题组 ${ }^{[115]}$ 也开展了一系列与该策 略相关的研究工作. 例如, 采用外消旋的 2-(三甲基甲硅 烷基)乙基亚砜前体 92 以及烯丙基氯化钯(II)二聚体 $[\mathrm{Pd}(\mathrm{allyl}) \mathrm{Cl}]_{2}$ 与 Josiphos 配体 101 催化体系 ${ }^{[113]}$, 经由 $\mathrm{CsF}$ 促进的消去反应原位生成次磺酸阴离子, 在比较温 和的条件下 $\left(40{ }^{\circ} \mathrm{C}\right)$, 实现了对它的不对称芳基化和烯 基化反应，从而将亲电试剂底物从溴代芳烃扩展至溴代 烯烃 96b (Scheme 14b). 此外, 2018 年, Bolm 课题组 ${ }^{[116]}$ 采用 $\mathrm{CuBr}_{2}$ 和 2,2'-联吡啶配体以及过氧化苯甲酸叔丁酯 $(\mathrm{BzOO} t-\mathrm{Bu})$ 氧化剂, 对茮位 $\mathrm{C}_{\mathrm{sp} 3}-\mathrm{H}$ 键进行氧化活化后, 再与原位生成的次磺酸阴离子偶联得到亚砜, 产率高达 $88 \%$, 为后续进一步开发手性廉价金属催化剂体系应用 于手性亚砜合成提供了支撑.

除了过渡金属催化剂体系, Perrio 课题组也将金鸡 纳生物碱季铵盐类手性相转移催化剂 105 应用于此类反 应之中, 通过对次磺酸阴离子的烷基化, 得到了具有中 等对映选择性的亚砜产物(58\%ee) (Scheme 15a) ${ }^{[111,120]}$. 2014 年, Tan 课题组 ${ }^{[121]}$ 开发了一种具有新颖结构的 “盘 扭五氮胍盐” 双功能相转移催化剂 106 (Scheme 15b), 通过离子对作用和卤键作用分别稳定次磺酸阴离子中 间体和活化卤代烷烃亲电试剂，大大提高了反应的对映 选择性(71\% 99\%ee 和底物范围. 近年来, Bolm, Waser 和 Zhang 课题组相继发展了无过渡金属催化剂参 与的偶联反应体系(Scheme 13), 采用二芳基碘鎓盐芳 基化试剂 98 ${ }^{[117,122]}$ 或基于 Ethynyl benziodoxolone (EBX) 的炔基化试剂 $99^{[118]}$ 来捕获原位生成的次磺酸阴离子中 


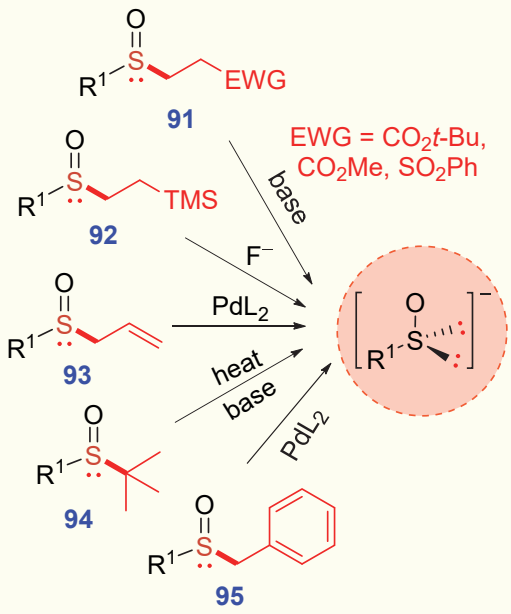

$\mathrm{R}^{1}=$ aryl, heteroaryl, benzyl, alkyl
$\mathrm{R}^{2}-\mathrm{X}$

$96 \mathrm{R}^{2}=$ aryl, heteroaryl, allyl, alkenyl, benzyl, alkyl; $\mathrm{X}=\mathrm{Br}, \mathrm{I}, \mathrm{OAC}$

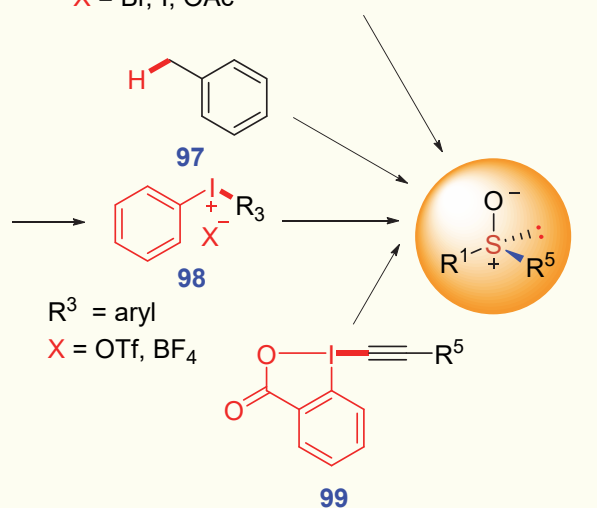

$\mathrm{R}^{5}=$ aryl, heteroaryl, alkenyl, benzyl, alkyl, alkynyl

图式 13 经由次磺酸阴离子合成手性亚砜

Scheme 13 Approaches to sulfenate anion towards sulfoxide synthesis

(a)

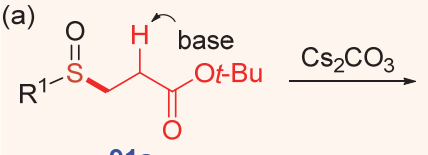
$91 \mathrm{a}$<smiles>[R]OC(=O)CC=[SH](=O)OCC</smiles>

I

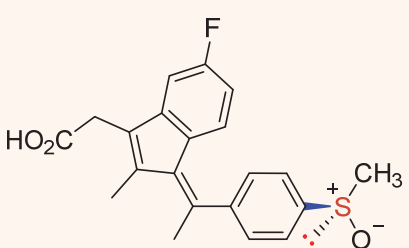

116 examples

(R)-Sulindac 52 $77 \%$ yield, $93 \%$ ee<smiles>C1CCC2C3CC(CN4CCCCN4C3)C2C1</smiles>

Ligand 100 (-)-Sparteine

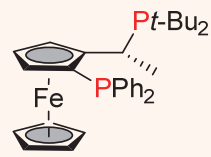

Ligand 101 Josiphos
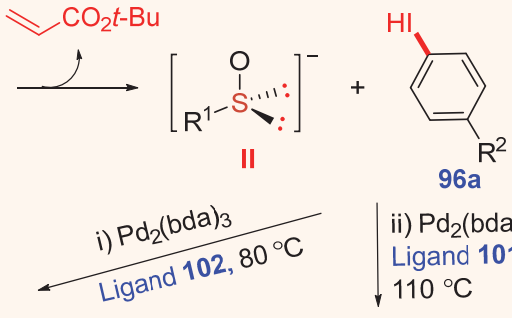

ii) $\mathrm{Pd}_{2}(\mathrm{bda})_{3}$ Ligand 101 $110{ }^{\circ} \mathrm{C}$

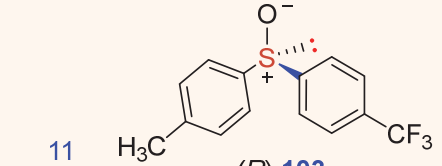

(R)-103

$98 \%$ yield, $80 \%$ ee

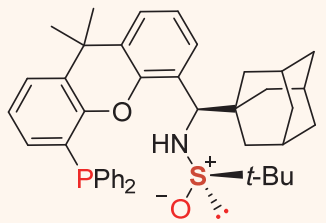

O,P-Ligand 102

(b)

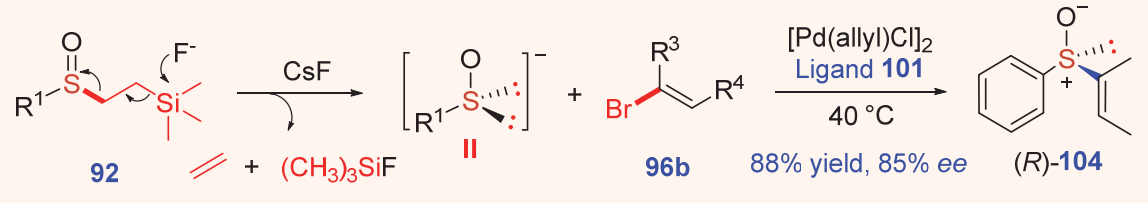

图式 $14 \mathrm{Pd}$ 催化的不对称芳基化和烯基化反应

Scheme 14 Arylation/alkenylation of sulfenate anion catalyzed by Pd complex catalysts

间体，高效地构建了具有结构多样性的亚砜化合物，为 下一步开发合成手性亚砜的反应体系奠定了基础.

综上所述，运用次磺酸阴离子中间体策略，通过构 建 $\mathrm{C}-\mathrm{S}$ 键, 能够高产率、高对映选择性地得到手性亚 砜, 且具有底物普适性广和官能团兼容性好等特点. 因 此, 该策略是对 Anderson 法和硫醚氧化法的重要补充,
能够避免使用化学当量的手性辅剂以及砜过氧化副产 物的生成. 但是, 该策略无法合成高光学活性的双烷基 亚砜, 且目前仍处于实验室研究阶段, 需要进一步设计 实用的手性催化体系来促进其工业化应用进程.

\section{7 其他策略}

以无光学活性的(非手性或者外消旋)亚砜作为反应 
a)
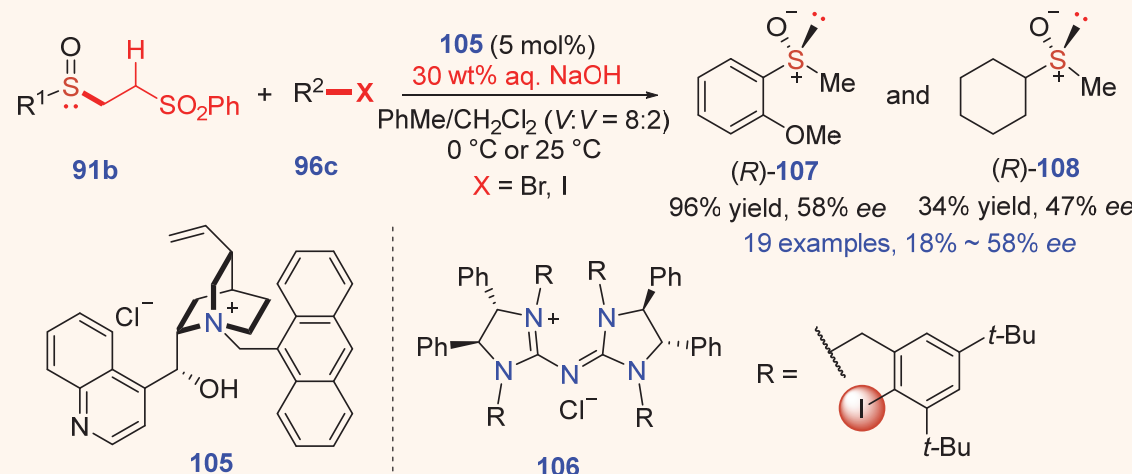

Cinchonidinium

b)

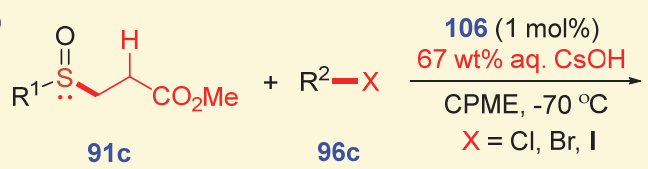<smiles>[R]C1C(/N=C2/[C@@H](c3ccccc3)[N+]([R])C(c3ccccc3)N2[R])N([R])C(c2ccccc2)N1[R]</smiles>

106 Pentanidinium $96 \%$ yield, $58 \%$ ee $34 \%$ yield, $47 \%$ ee 19 examples, $18 \% \sim 58 \%$ ee

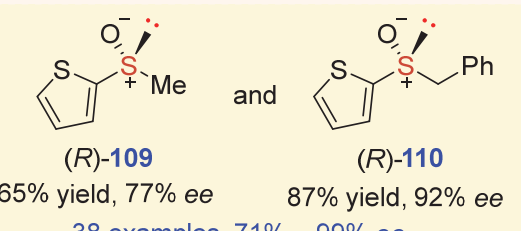

38 examples, $71 \% \sim 99 \%$ ee

图式 15 相转移催化的不对称烷基化反应

Scheme 15 Alkylation of sulfenate anion catalyzed by phase-transfer catalysis

底物, 采用还原动力学拆分、氧化动力学拆分、平行动 力学拆分、去对称化以及去外消旋化等策略(Scheme 16a)，也可以获得手性亚砜.

动力学拆分是获得手性化合物的最古老的方法之 一, 在手性试剂作用下, 外消旋混合物中两个对映体的 化学反应活性不再相同, 可利用两者反应速率的显著差 异(Schemes 16b, i), 进而分离纯化得到一个对映体, 其 产率不超过 $50 \%$. 其中选择性因数 $s=k_{S} / k_{R}$, 是判断手 性催化剂的动力学拆分能力的参数.

Gao 课题组 ${ }^{[123]}$ 以手性类卟啉配体 $\mathbf{1 1 1}$ 与锰(II) 原位 络合物为催化剂, 以 $\mathrm{H}_{2} \mathrm{O}_{2}$ 为氧化剂, 以金刚烷甲酸为添 加剂, 以外消旋芳基亚砜为底物, 采用氧化动力学拆分 方策略获得了高对映过量值的亚砜 69 以及具有实用价 值的砜 113 (Scheme 17a), 反应规模可达到克级, 具有 一定的实际应用意义, 且适用于外消旋杂芳基亚砜底 物. 之后, Gao 课题组采用此类锰络合物催化剂进行杂 芳基硫醚的不对称氧化, 可以直接得到结构多样的手性 亚砜产物 ${ }^{[124]}$. Míšek 课题组 ${ }^{[125]}$ 和 Yang 课题组 ${ }^{[126]}$ 采用蛋 氨酸亚砜还原酶 (MsrA) 或者二甲基亚砜还原酶 (DmsABC) 作为生物催化剂, 二硫苏糖醇(DTT)作为还 原剂, 在温和条件下选择性地还原 $(S)$-亚砜 115 为硫醚 $(s>100)$, 通过还原动力学拆分策略得到 $(R)$ - 亚砜 115 (Schemes 17b, i). 值得一提的是, Míšek 课题组 ${ }^{[125]}$ 采用 含有 MsrA 的大肠杆菌整细胞作为催化剂以及生物还原 体系, 在外加氧化剂条件下 114 使硫醚产物持续转化为 外消旋亚砜, 再进入到还原动力学拆分过程, 能够以高 达 90\%的产率获得光学纯的 $(R)$-亚砜 115 (Scheme 17b, ii). a) Miscellaneous strategies for chiral sulfoxide synthesis

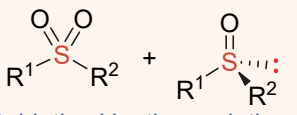

$$
\begin{aligned}
& R^{1-S_{1 . \cdots, 1}} R^{1}=R^{2} \\
& \text { Oxidative kinetic resolution a) a) Desymmetrization }
\end{aligned}
$$

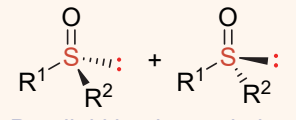

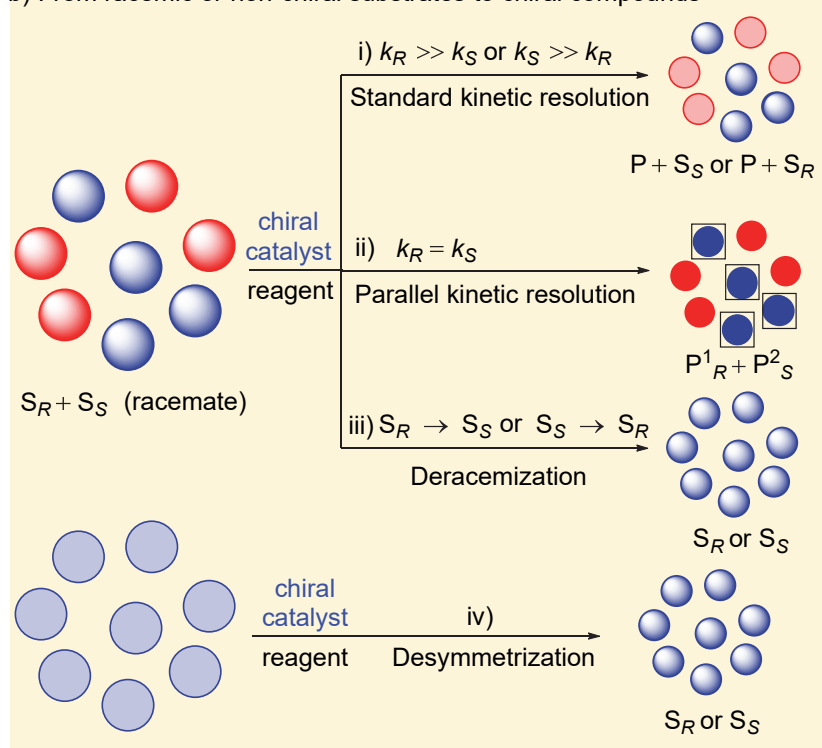

S non-chiral substate

图式 16 其他策略

Scheme 16 Miscellaneous strategies 
(a) Oxidative kinetic resolution

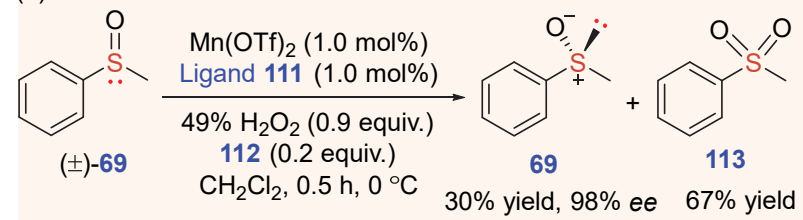

(hetero)aromatic sulfoxides: 35 examples,

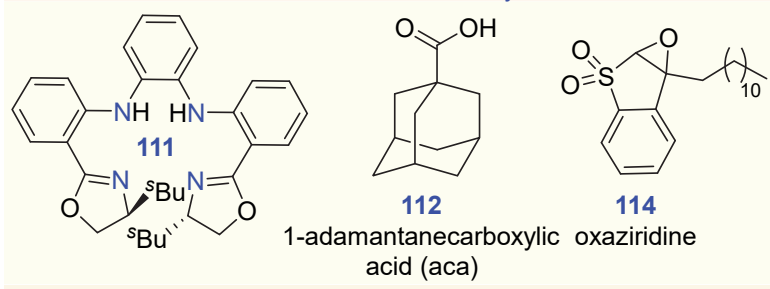

(b) Reductive kinetic resolution

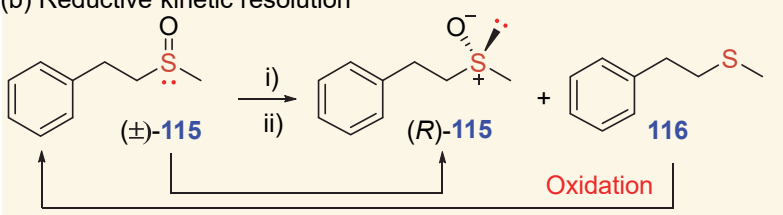

(i) MsrA (0.1 mol\%), $50 \mathrm{mmol} / \mathrm{L}$ PBS, pH 7.5, DTT ( 4 equiv.), $37^{\circ} \mathrm{C}, 5 \mathrm{~h}$

(ii) MsrA (0.1 mol\%) whole E. coli cells, buffer/decane, $114,37^{\circ} \mathrm{C}, 24 \mathrm{~h}$ aromatic sulfoxides: 12 examples, $52 \% \sim 90 \%$ yield, $97 \% \sim 99 \%$ ee

DTT = Dithiothreitol; PBS = phosphate-buffered saline

图式 17 动力学拆分策略

Scheme 17 Kinetic resolution of racemic sulfoxide

去对称化是以非光学活性分子为底物, 通过化学转 化打破分子的对称性, 进而将潜手性中心转化真正的立
体中心(Schemes 16b, iv). 平行动力学拆分策略是利用 两个对映体具有相同的反应速率却具有不同反应位点 的特点，给出易分离的反应产物(Schemes 16, ii). 2018 年, Wang 课题组 ${ }^{[127]}$ 报道了基于去对称化策略和平行动 力学拆分策略的 $\mathrm{Pd}(\mathrm{II})$ 催化芳基非手性亚砜或外消旋亚 砜的不对称 $\mathrm{C}_{\mathrm{sp} 2}-\mathrm{H}$ 烯烃化反应(Scheme 18a), 获得了优 异的对映选择性(高达 99\% ee), 为手性亚砜的合成打开 了一条新思路. 在此基础上, 2020 年, Wang 课题 组 ${ }^{[128]}$ 发展了 $\operatorname{Ir}(\mathrm{III})$ 催化的不对称 $\mathrm{C}_{\mathrm{sp}^{2}}-\mathrm{H}$ 酰胺化反应(Scheme $18 \mathrm{~b})$, 通过 $\mathrm{C}-\mathrm{N}$ 键的构建，以较高的收率 $(86 \%)$ 获得了 具有高对映过量值 $(98 \% e e)$ 的亚砜，证实了去对称化策 略和平行动力学拆分策略的实用性, 并且丰富了手性亚 砜配体的结构骨架.

去外消旋化是指在一定反应条件下，在降低外消旋 混合物中一种对映体数量的同时，能够增加另一种对映 体数量, 从而使外消旋体转化为单一对映体的过程 (Schemes 16b, iii). 然而, 因两种对映体的能量相同, 实 现这一过程面临着热力学上和动力学上两个方面的挑 战. 2019 年, Bach 课题组 ${ }^{[129]}$ 报道了在可见光条件下, 利 用手性三重态光敏剂 127 在 $\lambda=366 \mathrm{~nm}$ 实现了对基于内 酰胺骨架的亚砜 128 的部分去外消旋化(Scheme 19), 产 率为 $98 \%$, 对映过量值为 $55 \%$, 其中 $(S)-128$ 与光敏剂 127 的氢键作用更强, 具有更高的能量转移效率且易于

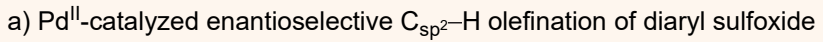

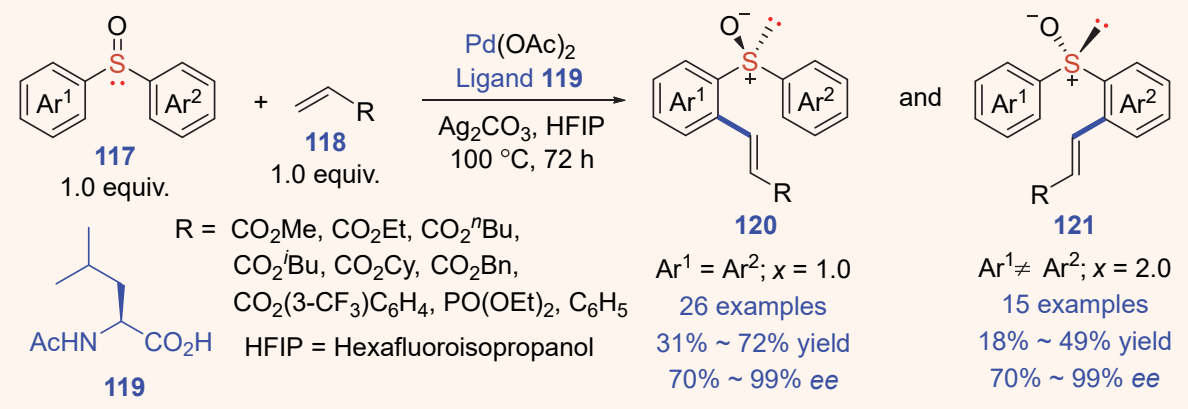

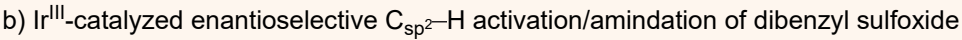

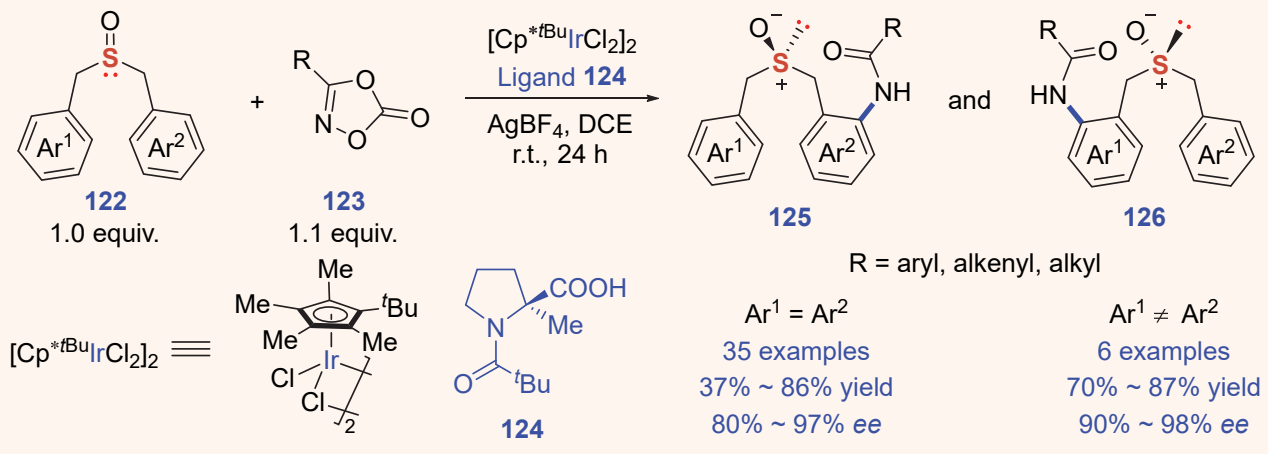

图式 18 去对称化和平行动力学拆分

Scheme 18 Desymmetrization and parallel kinetic resolution 


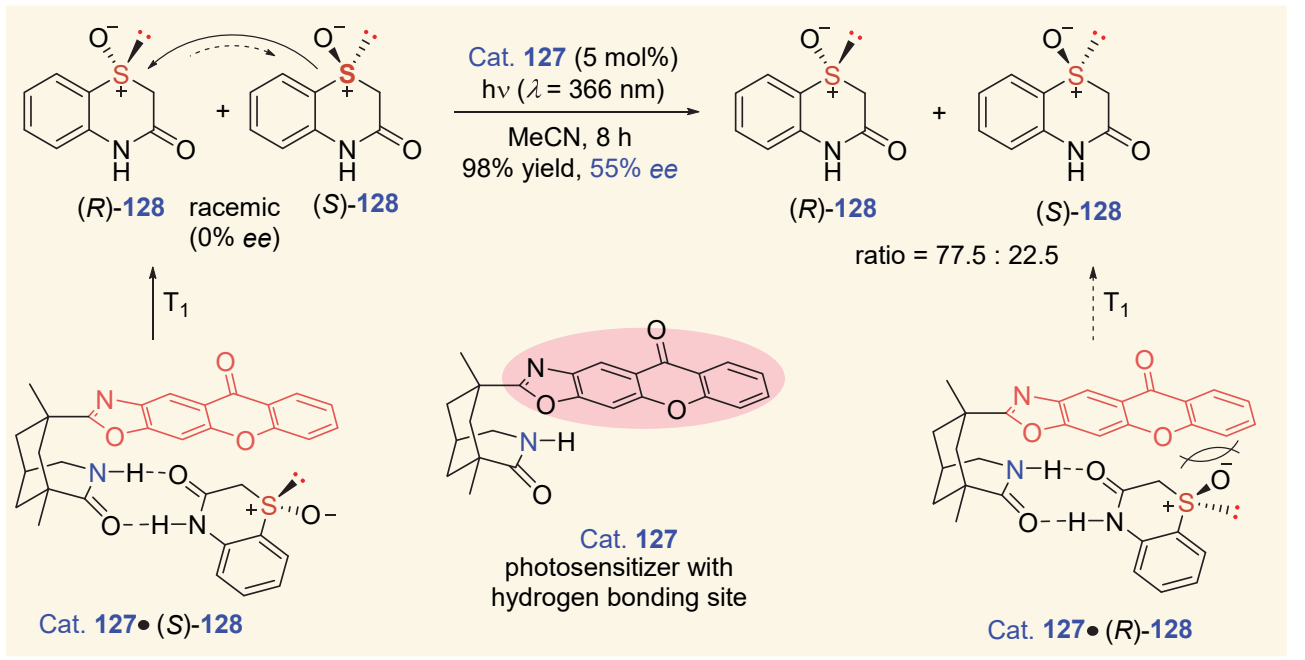

图式 19 光致去外消旋化策略

Scheme 19 Photochemical deracemization

发生立体翻转进而转化为 $(R)-\mathbf{1 2 8}$. 虽然目前底物范围 局限于 5 个例子且反应的对映选择性中等, 但随着手性 光催化剂的不断涌现, 该策略不失为一种制备手性亚砜 的新途径.

\section{3 总结和展望}

本文详细介绍了亚砜类化合物的生物活性以及相 应的结构骨架，从最具代表性的质子潅抑制剂奥美拉唑 切入讨论, 引入基于天然产物的阿焦烯、萝卜硫素、莱 菔素以及它们的衍生物, 接着阐述了 Cenicriviroc 的抗 HIV 病毒活性, 并对阿比多尔亚砜衍生物的抗 HBV 病 毒活性进行探讨, 着重阐明了阿莫达菲尼的药效作用机 制, 最后列举了其他一些具有抗炎、免疫抑制、神经保 护、抗精神病、抗耐药菌、降血脂、抗血栓等药物活性 的亚砜类化合物. 然而, 纵观很多亚砜类化合物的生物 活性研究, 只有极少数有明确的作用机制和清晰的构效 关系, 未来有望借助于先进的冷冻电镜结构解析技术、 化合物高通量篮选平台、高性能计算技术并结合系统的 药效动力学研究, 进行作用靶点验证, 为进一步结构改 造提供依据.

手性亚砜类化合物除了在手性药物市场占有一席 之位外, 也被广泛地应用于有机合成以及金属催化领 域, 同时增进了人们对其他含硫手性中心化合物, 如手 性亚砜亚胺 $(\mathrm{S}(\mathrm{O})(\mathrm{NR}))$ 以及手性亚磺酰胺 $(\mathrm{S}(\mathrm{O}) \mathrm{N})$ 的研 究兴趣. 本文在参考国内外相关文献的基础上, 从传统 Anderson 法和 Kagan-Moderna 法出发, 着重介绍了手性 亚砜合成领域的重要进展和新方法如去对称化策略, 也 包括作者采用次磺酸阴离子策略和多金属氧酸盐催化 体系进行亚砜不对称合成的研究工作. 次磺酸阴离子策 略与 Anderson 法的相似之处是两者均通过构建碳一硫键
来合成手性亚砜, 不同之处在于次磺酸阴离子中的硫原 子具有亲核性且为潜手性，而 Anderson 法采用的亚磺 酸酯具有亲电性且为单一非对映异构体. 采用次磺酸阴 离子策略制备手性亚砜，具有类似组合化学的特点，采 用 $m$ 个次磺酸阴离子前体模块与 $n$ 个亲电模块反应, 得 到 $m^{*} n$ 个产物，可通过快速构建手性亚砜化合物库助力 高通量篎选等药物研发. 价廉高效的铁金属络合物和多 金属氧酸盐催化剂，因反应条件温和且操作简单，适用 于手性亚砜的工业化生产. 采用无金属参与的有机小分 子催化剂以及生物催化、电催化体系进行硫醚不对称氧 化具有绿色化学的特点，而采用光致去消旋化策略制备 亚砜具有新颖性以及理论意义.

相信随着药物研发手段的不断进步和不对称催化 合成技术的日臻成熟，人们将会继续构建出结构多样的 含硫手性中心的化合物包括但不限于手性亚砜，进一步 扩宽其应用范围并发掘出更多的生物活性.

\section{References}

[1] Pattillo, C. C.; Strambeanu, I. I.; Calleja, P.; Vermeulen, N. A.; Mizuno, T.; White, M. C. J. Am. Chem. Soc. 2016, 138, 1265.

[2] Khiar, N.; Fernández, I.; Alcudia, F. Tetrahedron Lett. 1993, 34, 123.

[3] Xue, F.; Li, X.; Wan, B. J. Org. Chem. 2011, 76, 7256.

[4] Du, L.; Cao, P.; Xing, J.; Lou, Y.; Jiang, L.; Li, L.; Liao, J. Angew. Chem. Int. Ed. 2013, 52, 4207.

[5] James, B. R.; McMillan, R. S. Can. J. Chem. 1977, 55, 3927.

[6] Trost, B. M.; Rao, M. Angew. Chem. Int. Ed. 2015, 54, 5026.

[7] Jia, T.; Wang, M.; Liao, J. Top. Curr. Chem. 2019, 377, 8.

[8] Pulis, A. P.; Procter, D. J. Angew. Chem. Int. Ed. 2016, 55, 9842.

[9] Zeng, J.; Liu, Y.; Chen, W.; Zhao, X.; Meng, L.; Wan, Q. Top. Curr. Chem. 2018, 376, 27.

[10] Salom-Roig, X.; Bauder, C. Synthesis 2020, 52, 964.

[11] Chen, J.; Chen, J.; Lang, F.; Zhang, X.; Cun, L.; Zhu, J.; Deng, J.; Liao, J. J. Am. Chem. Soc. 2010, 132, 4552

[12] Calabro, K.; Jennings, L. K.; Lasserre, P.; Doohan, R.; Rodrigues, D.; Reyes, F.; Ramos, C.; Thomas, O. P. J. Org. Chem. 2020, 85, 
14026.

[13] Yuan, W. H.; Teng, M. T.; Yun, Y. F.; Jiang, N.; Ma, L.; Sun, S. S.; Yuan, B.; Tang, J.; Wu, Q. Y.; Li, Q.; Zhang, P.; Morris-Natschke, S. L.; Lee, K. H.; Talarolactone, A. J. Nat. Prod. 2020, 83, 1716.

[14] Liu, D. H.; Sun, Y. Z.; Kurtan, T.; Mandi, A.; Tang, H.; Li, J.; Su, L.; Zhuang, C. L.; Liu, Z. Y.; Zhang, W. J. Nat. Prod. 2019, 82, 1274.

[15] Chaudhary, N. K.; Pitt, J. I.; Lacey, E.; Crombie, A.; Vuong, D.; Piggott, A. M.; Karuso, P. J. Nat. Prod. 2018, 81, 1517.

[16] Lutz, C.; Simon, W.; Werner-Simon, S.; Pahl, A.; Muller, C. Angew. Chem. Int. Ed. 2020, 59, 11390 .

[17] Matinkhoo, K.; Pryyma, A.; Todorovic, M.; Patrick, B. O.; Perrin, D. M. J. Am. Chem. Soc. 2018, 140, 6513 .

[18] Siegert, M. J.; Knittel, C. H.; Sussmuth, R. D. Angew. Chem. Int. Ed. 2020, 59, 5500

[19] Surur, A. S.; Schulig, L.; Link, A. Arch. Pharm. 2019, 352, 1800248.

[20] Rafique, W.; Kramer, V.; Pardo, T.; Smits, R.; Spilhaug, M. M.; Hoepping, A.; Savio, E.; Engler, H.; Kuljs, R.; Amaral, H.; Riss, P. J. Acs Omega 2018, 3, 7567.

[21] Block, E.; Ahmad, S.; Jain, M. K.; Crecely, R. W.; Apitz-Castro, R.; Cruz, M. R. J. Am. Chem. Soc. 1984, 106, 8295.

[22] Fong, J.; Yuan, M.; Jakobsen, T. H.; Mortensen, K. T.; Delos Santos, M. M. S.; Chua, S. L.; Yang, L.; Tan, C. H.; Nielsen, T. E.; Givskov, M. J. Med. Chem. 2017, 60, 215.

[23] Silva, F.; Khokhar, S. S.; Williams, D. M.; Saunders, R.; Evans, G. J. S.; Graz, M.; Wirth, T. Angew. Chem. Int. Ed. 2018, 57, 12290.

[24] Raynbird, M. Y.; Silva, F.; Smallman, H.; Khokhar, S. S.; Neef, D.; Evans, G. J. S.; Wirth, T. Chem. Eur. J. 2020, 26, 8363.

[25] Kaschula, C. H.; Hunter, R.; Stellenboom, N.; Caira, M. R.; Winks, S.; Ogunleye, T.; Richards, P.; Cotton, J.; Zilbeyaz, K.; Wang, Y.; Siyo, V.; Ngarande, E.; Parker, M. I. Eur. J. Med. Chem. 2012, 50, 236.

[26] Stellenboom, N. J. Turk. Chem. Soc., Sect. A 2019, 6, 143.

[27] Cuomo, V.; Luciano, F. B.; Meca, G.; Ritieni, A.; Mañes, J. CyTA-J. Food 2015, 13, 361.

[28] Nastruzzi, C.; Cortesi, R.; Esposito, E.; Menegatti, E.; Leoni, O.; Iori, R.; Palmieri, S. J. Agric. Food Chem. 1996, 44, 1014.

[29] Fahey, J. W.; Holtzclaw, W. D.; Wehage, S. L.; Wade, K. L.; Stephenson, K. K.; Talalay, P. PLoS One 2015, 10, e0140963.

[30] Parfenova, H.; Liu, J.; Hoover, D. T.; Fedinec, A. L. J. Cereb. Blood Flow Metab. 2019, 40, 1987.

[31] Tarozzi, A.; Angeloni, C.; Malaguti, M.; Morroni, F.; Hrelia, S.; Hrelia, P. Oxid. Med. Cell. Longevity 2013, 2013, 415078.

[32] Kakizaki, T.; Kitashiba, H.; Zou, Z.; Li, F.; Fukino, N.; Ohara, T.; Nishio, T.; Ishida, M. Plant Physiol. 2017, 173, 1583.

[33] Wang, H.; Wang, F.; Wu, S.; Liu, Z.; Li, T.; Mao, L.; Zhang, J.; Li, C.; Liu, C.; Yang, Y. Chem. Biol. Interact. 2018, 281, 11.

[34] Tian, X.; Gao, J.; Liu, M.; Lei, Y.; Wang, F.; Chen, J.; Chu, P.; Gao, J.; Long, F.; Liang, M.; Long, X.; Chu, H.; Liu, C.; Li, X.; Sun, Q.; Li, G.; Yang, Y. J. Med. Chem. 2020, 63, 3881.

[35] Ning, X.; Guo, Y.; Wang, X.; Ma, X.; Tian, C.; Shi, X.; Zhu, R.; Cheng, C.; Du, Y.; Ma, Z.; Zhang, Z.; Liu, J. J. Med. Chem. 2014, 57, 4302 .

[36] Ning, X.; Yuan, M.; Guo, Y.; Tian, C.; Wang, X.; Zhang, Z.; Liu, J. J. Enzyme Inhib. Med. Chem. 2016, 31, 464.

[37] Seto, M.; Aikawa, K.; Miyamoto, N.; Aramaki, Y.; Kanzaki, N.; Takashima, K.; Kuze, Y.; Iizawa, Y.; Baba, M.; Shiraishi, M. J. Med. Chem. 2006, 49, 2037.

[38] Song, S.; Tian, Q.; Wu, Y.; Zhao, M.; Wang, C.; Cai, J.; Wang, L.; Wang, J. WO 2019149089, 2019.

[39] Luo, Y.; Long, C.; Ba, Y.; Chen, X.; Chen, S. WO 2018103757, 2018.

[40] Grinev, A. N.; Pershin, G. N. WO 9008135, 1990

[41] Leneva, I. A. WO 2007075102, 2007.

[42] Chai, H.; Zhao, Y.; Zhao, C.; Gong, P. Biorg. Med. Chem. 2006, 14, 911.

[43] Chai, H.-F.; Liang, X.-X.; Li, L.; Zhao, C.-S.; Gong, P.; Liang,
Z.-J.; Zhu, W.-L.; Jiang, H.-L.; Luo, C. J. Mol. Model. 2011, 17, 1831.

[44] Ivashchenko, A. V.; Yamanushkin, P. M.; Mit'kin, O. D.; Kisil, V. M.; Korzinov, O. M.; Vedenskii, V. Y.; Leneva, I. A.; Bulanova, E. A.; Bychko, V. V.; Okun, I. M. Pharm. Chem. J. 2014, 47, 636.

[45] Di Mola, A.; Peduto, A.; La Gatta, A.; Delang, L.; Pastorino, B.; Neyts, J.; Leyssen, P.; de Rosa, M.; Filosa, R. Bioorg. Med. Chem. 2014, 22, 6014.

[46] Scuotto, M.; Abdelnabi, R.; Collarile, S.; Schiraldi, C.; Delang, L.; Massa, A.; Ferla, S.; Brancale, A.; Leyssen, P.; Neyts, J.; Filosa, R. Bioorg. Med. Chem. 2017, 25, 327.

[47] Gerrard, P.; Malcolm, R. Neuropsychiatr. Dis. Treat. 2007, 3, 349.

[48] Loland, C. J.; Mereu, M.; Okunola, O. M.; Cao, J.; Prisinzano, T. E.; Mazier, S.; Kopajtic, T.; Shi, L.; Katz, J. L.; Tanda, G.; Newman, A. H. Biol. Psychiatry. 2012, 72, 405.

[49] Abramyan, A. M.; Stolzenberg, S.; Li, Z.; Loland, C. J.; Noé, F.; Shi, L. ACS Chem. Neurosci. 2017, 8, 1735.

[50] Cao, J.; Slack, R. D.; Bakare, O. M.; Burzynski, C.; Rais, R.; Slusher, B. S.; Kopajtic, T.; Bonifazi, A.; Ellenberger, M. P.; Yano, H.; He, Y.; Bi, G.-H.; Xi, Z.-X.; Loland, C. J.; Newman, A. H. J. Med. Chem. 2016, 59, 10676.

[51] Rotolo, R. A.; Dragacevic, V.; Kalaba, P.; Urban, E.; Zehl, M.; Roller, A.; Wackerlig, J.; Langer, T.; Pistis, M.; De Luca, M. A.; Caria, F.; Schwartz, R.; Presby, R. E.; Yang, J.-H.; Samels, S.; Correa, M.; Lubec, G.; Salamone, J. D. Front Pharmacol. 2019, 10.

[52] Kalaba, P.; Ilić, M.; Aher, N. Y.; Dragačević, V.; Wieder, M.; Zehl, M.; Wackerlig, J.; Beyl, S.; Sartori, S. B.; Ebner, K.; Roller, A.; Lukic, N.; Beryozkina, T.; Gonzalez, E. R. P.; Neill, P.; Khan, J. A.; Bakulev, V.; Leban, J. J.; Hering, S.; Pifl, C.; Singewald, N.; Lubec, J.; Urban, E.; Sitte, H. H.; Langer, T.; Lubec, G. J. Med. Chem. 2020, 63, 391.

[53] Suh, S.-H.; Kim, S.-J. WO 2020055166, 2020.

[54] Goundry, W. R. F.; Dai, K.; Gonzalez, M.; Legg, D.; O'KearneyMcMullan, A.; Morrison, J.; Stark, A.; Siedlecki, P.; Tomlin, P.; Yang, J. Org. Process Res. Dev. 2019, 23, 1333.

[55] Dillon, M. T.; Bergerhoff, K. F.; Pedersen, M.; Whittock, H.; Crespo-Rodriguez, E.; Patin, E. C.; Pearson, A.; Smith, H. G.; Paget, J. T. E.; Patel, R. R.; Foo, S.; Bozhanova, G.; Ragulan, C.; Fontana, E.; Desai, K.; Wilkins, A. C.; Sadanandam, A.; Melcher, A.; McLaughlin, M.; Harrington, K. J. Clin. Cancer Res. 2019, 25, 3392.

[56] Di Carlo, F. J. Drug Metab. Rev. 1979, 10, 225.

[57] Freedman, H. H.; Fox, A. E.; Shavel, J.; Morrison, G. C. Proc. Soc. Exp. Biol. Med. 1972, 139, 909.

[58] Saitoh, M.; Kunitomo, J.; Kimura, E.; Iwashita, H.; Uno, Y.; Onishi, T.; Uchiyama, N.; Kawamoto, T.; Tanaka, T.; Mol, C. D.; Dougan, D. R.; Textor, G. P.; Snell, G. P.; Takizawa, M.; Itoh, F.; Kori, M. J. Med. Chem. 2009, 52, 6270.

[59] Hosie, A. M.; Baylis, H. A.; Buckingham, S. D.; Sattelle, D. B. Br. J. Pharmacol. 1995, 115, 909.

[60] Klimas, M. T.; Goldstein, J. M.; Trainor, D. A.; Jacobs, R. T.; Ohnmacht, C. J.; Roberts, R. A.; Yee, Y. K.; Terpko, M. O.; Thomas, S. P.; Cronk, L. A.; Frank, C. A.; Harris, G. D.; Hulsizer, J.; Lewis, J. J.; McLaren, F. M.; Mauger, R. C.; Morosky, G. D.; Ronkin, S. M.; Sienkewicz, P.; Sparks, R. B.; Ulatowski, T. G.; Wildonger, D. Bioorg. Med. Chem. Lett. 1995, 5, 1795.

[61] Hogan, P. J.; Hopes, P. A.; Moss, W. O.; Robinson, G. E.; Patel, I. Org. Process Res. Dev. 2002, 6, 225.

[62] Salillas, S.; Alías, M.; Michel, V.; Mahía, A.; Lucía, A.; Rodrigues, L.; Bueno, J.; Galano-Frutos, J. J.; De Reuse, H.; VelázquezCampoy, A.; Carrodeguas, J. A.; Sostres, C.; Castillo, J.; Aínsa, J. A.; Díaz-de-Villegas, M. D.; Lanas, Á.; Touati, E.; Sancho, J. J. Med. Chem. 2019, 62, 6102 .

[63] Pitchen, P.; France, C. J.; McFarlane, I. M.; Newton, C. G.; Thompson, D. M. Tetrahedron Lett 1994, 35, 485.

[64] Smith, C.; Ashton, M. J.; Bush, R. C.; Facchini, V.; Harris, N. V.; Hart, T. W.; Jordan, R.; MacKenzie, R.; Riddell, D. Bioorg. Med. Chem. Lett. 1996, 6, 47. 
[65] Riddell, D.; Bright, C. P.; Burton, B. J.; Bush, R. C.; Harris, N. V.; Hele, D.; Moore, U. M.; Naik, K.; Parrott, D. P.; Smith, C.; Williams, R. J. Biochem. Pharmacol. 1996, 52, 1177.

[66] Ozeki, Y.; Nagamura, Y.; Ito, H.; Unemi, F.; Kimura, Y.; Igawa, T.; Kambayashi, J.-i.; Takahashi, Y.; Yoshimoto, T. Br. J. Pharmacol. 1999, 128, 1699.

[67] Uno, T.; Ozeki, Y.; Koga, Y.; Chu, G.-N.; Okada, M.; Tamura, K.; Igawa, T.; Unemi, F.; Kido, M.; Nishi, T. Chem. Pharm. Bull. 1995, 43, 1724.

[68] Yoneda, T.; Tabata, H.; Tasaka, T.; Oshitari, T.; Takahashi, H.; Natsugari, H. J. Med. Chem. 2015, 58, 3268.

[69] Makino, K.; Yoneda, T.; Ogawa, R.; Kanase, Y.; Tabata, H.; Oshitari, T.; Natsugari, H.; Takahashi, H. Tetrahedron Lett 2017, 58, 2885.

[70] Kubota, H.; Kakefuda, A.; Nagaoka, H.; Yamamoto, O.; Ikeda, K.; Takeuchi, M.; Shibanuma, T.; Isomura, Y. Chem. Pharm. Bull. 1998, 46, 242.

[71] Rodriguez, S.; Haddad, N.; Frutos, R. P.; Grinberg, N.; Krishnamurthy, D.; Senanayake, C. H. Org. Process Res. Dev. 2017, 21, 444.

[72] Boehringer Ingelheim Pharma GmbH \& Co. KG EP1847543, 2007.

[73] Harrison, P. W. B.; Kenyon, J.; Phillips, H. J. Chem. Soc. 1926, 129, 2079.

[74] Andersen, K. K. Tetrahedron Lett. 1962, 3, 93.

[75] Pitchen, P.; Kagan, H. B. Tetrahedron Lett. 1984, 25, 1049.

[76] Pitchen, P.; Dunach, E.; Deshmukh, M. N.; Kagan, H. B. J. Am. Chem. Soc. 1984, 106, 8188.

[77] Di Furia, F.; Modena, G.; Seraglia, R. Synthesis 1984, 325.

[78] Zeng, Q. L. Prog. Chem. 2007, 19, 745 (in Chinese). (曾庆乐, 化学进展, 2007, 19, 745.)

[79] Srour, H.; Le Maux, P.; Chevance, S.; Simonneaux, G. Coord. Chem. Rev. 2013, 257, 3030.

[80] Wojaczyńska, E.; Wojaczyński, J. Chem. Rev. 2010, 110, 4303.

[81] Wojaczyńska, E.; Wojaczyński, J. Chem. Rev. 2020, 120, 4578.

[82] Casnati, A.; Lanzi, M.; Cera, G. Molecules 2020, 25.

[83] Legros, J.; Bolm, C. Angew. Chem. Int. Ed. 2004, 43, 4225.

[84] Wang, F.; Feng, L.; Dong, S.; Liu, X.; Feng, X. Chem. Commun. 2020, 56, 3233 .

[85] Egami, H.; Katsuki, T. J. Am. Chem. Soc. 2007, 129, 8940.

[86] Le Maux, P.; Simonneaux, G. Chem. Commun. 2011, 47, 6957.

[87] Salles, L.; Robert, F.; Semmer, V.; Jeannin, Y.; Bregeault, J. M. Bull. Soc. Chim. Fr. 1996, 133, 319.

[88] Zuwei, X.; Ning, Z.; Yu, S.; Kunlan, L. Science 2001, 292, 1139.

[89] Chakravarthy, R. D.; Ramkumar, V.; Chand, D. K. Green Chem. 2014, 16, 2190 .

[90] Sato, K.; Hyodo, M.; Aoki, M.; Zheng, X.-Q.; Noyori, R. Tetrahedron 2001, 57, 2469.

[91] Jahier, C.; Coustou, M.-F.; Cantuel, M.; McClenaghan, N. D.; Buffeteau, T.; Cavagnat, D.; Carraro, M.; Nlate, S. Eur. J. Inorg. Chem. 2011, 2011, 727.

[92] Jahier, C.; Touzani, R.; El Kadiri, S.; Nlate, S. Inorg. Chim. Acta 2016, 450, 81

[93] Zong, L.; Wang, C.; Moeljadi, A. M.; Ye, X.; Ganguly, R.; Li, Y.; Hirao, H.; Tan, C. H. Nat. Commun. 2016, 7, 13455.

[94] Ye, X. Y.; Moeljadi, A. M. P.; Chin, K. F.; Hirao, H.; Zong, L. L.; Tan, C. H. Angew. Chem. Int. Ed. 2016, 55, 7101.

[95] Colonna, S.; Pironti, V.; Drabowicz, J.; Brebion, F.; Fensterbank, L.; Malacria, M. Eur. J. Org. Chem. 2005, 2005, 1727.

[96] Mojr, V.; Herzig, V.; Buděšínský, M.; Cibulka, R.; Kraus, T. Chem.
Commun. 2010, 46, 7599.

[97] Liu, Z.-M.; Zhao, H.; Li, M.-Q.; Lan, Y.-B.; Yao, Q.-B.; Tao, J.-C.; Wang, X.-W. Adv. Synth. Catal. 2012, 354, 1012.

[98] Liao, S.; Čorić, I.; Wang, Q.; List, B. J. Am. Chem. Soc. 2012, 134, 10765.

[99] Zhang, S.; Li, G.; Li, L.; Deng, X.; Zhao, G.; Cui, X.; Tang, Z. ACS Catal. 2020, 10, 245.

[100] Maczka, W.; Winska, K.; Grabarczyk, M. Catalysts 2018, 8, 27.

[101] Ren, S.-M.; Liu, F.; Wu, Y.-Q.; Chen, Q.; Zhang, Z.-J.; Yu, H.-L.; $\mathrm{Xu}$, J.-H. Biotechnol. Bioeng. 2021, 118: 737.

[102] Xu, N.; Zhu, J.; Wu, Y.-Q.; Zhang, Y.; Xia, J.-Y.; Zhao, Q.; Lin, G.-Q.; Yu, H.-L.; Xu, J.-H. Org. Process Res. Dev. 2020, 24, 1124.

[103] Chang, X. H.; Zhang, Q. L.; Guo, C. Angew. Chem. Int. Ed. 2020, 59, 12612 .

[104] Yan, M.; Kawamata, Y.; Baran, P. S. Chem. Rev. 2017, 117, 13230.

[105] Ghosh, M.; Shinde, V. S.; Rueping, M. Beilstein J. Org. Chem. 2019, 15, 2710.

[106] Firth, B. E.; Miller, L. L.; Mitani, M.; Rogers, T.; Lennox, J.; Murray, R. W. J. Am. Chem. Soc. 1976, 98, 8271.

[107] Komori, T.; Nonaka, T. J. Am. Chem. Soc. 1984, 106, 2656.

[108] O'Donnell, J. S.; Schwan, A. L. J. Sulfur Chem. 2004, 25, 183.

[109] Caupène, C.; Boudou, C.; Perrio, S.; Metzner, P. J. Org. Chem. 2005, 70, 2812.

[110] Maitro, G.; Vogel, S.; Sadaoui, M.; Prestat, G.; Madec, D.; Poli, G. Org. Lett. 2007, 9, 5493.

[111] Gelat, F.; Jayashankaran, J.; Lohier, J.-F.; Gaumont, A.-C.; Perrio, S. Org. Lett. 2011, 13, 3170.

[112] Gelat, F.; Lohier, J.-F.; Gaumont, A.-C.; Perrio, S. Adv. Synth Catal. 2015, 357, 2011.

[113] Wu, C.; Berritt, S.; Liang, X.; Walsh, P. J. Org. Lett. 2019, 21, 960.

[114] Bernoud, E.; Le Duc, G.; Bantreil, X.; Prestat, G.; Madec, D.; Poli, G. Org. Lett. 2010, 12, 320 .

[115] Jia, T.; Zhang, M.; McCollom, S. P.; Bellomo, A.; Montel, S.; Mao, J.; Dreher, S. D.; Welch, C. J.; Regalado, E. L.; Williamson, R. T.; Manor, B. C.; Tomson, N. C.; Walsh, P. J. Am. Chem. Soc. 2017, 139, 8337.

[116] Yu, H.; Li, Z.; Bolm, C. Org. Lett. 2018, 20, 2076.

[117] Wang, L.; Chen, M.; Zhang, J. Org. Chem. Front. 2019, 6, 32.

[118] Amos, S. G. E.; Nicolai, S.; Gagnebin, A.; Le Vaillant, F.; Waser, J. J. Org. Chem. 2019, 84, 3687.

[119] Wang, L.; Chen, M.; Zhang, P.; Li, W.; Zhang, J. J. Am. Chem. Soc. 2018, 140, 3467.

[120] Gelat, F.; Gaumont, A.-C.; Perrio, S. J. Sulfur Chem. 2013, 34, 596.

[121] Zong, L. L.; Ban, X.; Kee, C. W.; Tan, C. H. Angew. Chem. Int. Ed. 2014, 53, 11849 .

[122] Yu, H.; Li, Z.; Bolm, C. Org. Lett. 2018, 20, 7104.

[123] Yang, J. C.; Li, G. S.; Lu, C. W.; An, Y.; Gao, S. Chin. J. Org. Chem. 2018, 38, 3070.

[124] Dai, W.; Shang, S.; Lv, Y.; Li, G.; Li, C.; Gao, S. ACS Catal. 2017, 7, 4890.

[125] Nosek, V.; Misek, J. Angew. Chem. Int. Ed. 2018, 57, 9849.

[126] Peng, L. T.; Wen, Y. M.; Chen, Y.; Yuan, Z. M.; Zhou, Y.; Cheng, X. L.; Chen, Y. Z.; Yang, J. W. ChemCatChem 2018, 10, 3284.

[127] Zhu, Y.-C.; Li, Y.; Zhang, B.-C.; Zhang, F.-X.; Yang, Y.-N.; Wang, X.-S. Angew. Chem. Int. Ed. 2018, 57, 5129.

[128] Liu, W.; Yang, W.; Zhu, J.; Guo, Y.; Wang, N.; Ke, J.; Yu, P.; He, C. ACS Catal. 2020, 10, 7207.

[129] Wimberger, L.; Kratz, T.; Bach, T. Synthesis 2019, 51, 4417. 